\title{
Folate cycle enzyme MTHFD1L confers metabolic advantages in hepatocellular carcinoma
}

\author{
Derek Lee, ${ }^{1}$ Iris Ming-Jing Xu, ${ }^{1}$ David Kung-Chun Chiu, ${ }^{1}$ Robin Kit-Ho Lai, ${ }^{1}$ Aki Pui-Wah Tse, ${ }^{1}$ Lynna Lan Li, ${ }^{1}$ Cheuk-Ting Law, ${ }^{1}$ \\ Felice Ho-Ching Tsang, ${ }^{1}$ Larry Lai Wei, ${ }^{1}$ Cerise Yuen-Ki Chan, ${ }^{1}$ Chun-Ming Wong, ${ }^{1,2}$ Irene Oi-Lin Ng, ${ }^{1,2}$ and Carmen Chak-Lui Wong ${ }^{1,2}$ \\ 'Department of Pathology and 'State Key Laboratory for Liver Research, The University of Hong Kong, Hong Kong, China.
}

\begin{abstract}
Cancer cells preferentially utilize glucose and glutamine, which provide macromolecules and antioxidants that sustain rapid cell division. Metabolic reprogramming in cancer drives an increased glycolytic rate that supports maximal production of these nutrients. The folate cycle, through transfer of a carbon unit between tetrahydrofolate and its derivatives in the cytoplasmic and mitochondrial compartments, produces other metabolites that are essential for cell growth, including nucleotides, methionine, and the antioxidant NADPH. Here, using hepatocellular carcinoma (HCC) as a cancer model, we have observed a reduction in growth rate upon withdrawal of folate. We found that an enzyme in the folate cycle, methylenetetrahydrofolate dehydrogenase 1-like (MTHFD1L), plays an essential role in support of cancer growth. We determined that MTHFD1L is transcriptionally activated by NRF2, a master regulator of redox homeostasis. Our observations further suggest that MTHFD1L contributes to the production and accumulation of NADPH to levels that are sufficient to combat oxidative stress in cancer cells. The elevation of oxidative stress through MTHFD1L knockdown or the use of methotrexate, an antifolate drug, sensitizes cancer cells to sorafenib, a targeted therapy for HCC. Taken together, our study identifies MTHFD1L in the folate cycle as an important metabolic pathway in cancer cells with the potential for therapeutic targeting.
\end{abstract}

\section{Introduction}

Cancer cells exhibit at least 2 distinctive metabolic traits: increased glycolytic rate, and increased capacity against oxidative stress. Cancer cells, even in the presence of $\mathrm{O}_{2}$, preferentially utilize glycolytic fermentation to generate energy, a phenomenon known as the Warburg effect (1). Although less energy efficient, aerobic glycolysis coordinates other metabolic pathways for maximal production of macromolecules and antioxidants. One may ask why more macromolecules are needed by cancer cells. The answer lies in the fact that cancer cells need nucleotides for DNA synthesis and lipids for membrane synthesis to sustain rapid division. Similarly, one may also ask why antioxidants are needed by cancer cells. And this is due to cancer cells experiencing increased oxidative stress caused by various factors such as hypoxia, mitochondrial mutations, and aberrant signaling pathways that cause activated metabolic machineries (2). Low levels of ROS act to signal the activation of oncogenic pathways such as MAPK, ERK, JNK, Akt, and HIF (3). Low levels of ROS also promote DNA mutations and genomic instability, supporting transformation (4). Conversely, high levels of ROS irreversibly damage cellular components, causing cell cycle arrest and apoptosis (3). Cancer cells need higher antioxidant-producing capacity that enables them to survive oxidative stress $(5,6)$. Many conventional chemotherapies and radiotherapies eradicate cancer cells through ROS induction (7).

Authorship note: D. Lee, I.M.J. Xu, and D.K.C. Chiu contributed equally to this work. Conflict of interest: The authors have declared that no conflict of interest exists. Submitted: August 23, 2016; Accepted: February 16, 2017.

Reference information: / Clin Invest. 2017;127(5):1856-1872.

https://doi.org/10.1172/JCI90253.
The folate cycle is an important metabolic pathway that fulfills a number of cancer-specific nutrient demands. Folate (folic acid), or vitamin B, is commonly found in Western diets and dietary supplements. A 1-carbon (1C) unit from serine is transferred to tetrahydrofolate (THF) by serine hydroxymethyl transferases (SHMTs) to form 5,10-methylenetetrahydrofolate $\left(\mathrm{CH}_{2}\right.$ $\mathrm{THF}$ ). The $1 \mathrm{C}$ unit is then transferred from one position of THF to another, thus creating the folate cycle (Figure 1). The folate cycle is composed of the cytoplasmic and mitochondrial compartments. The cytoplasmic compartment is carried out by methylenetetrahydrofolate dehydrogenase, cyclohydrolase, and formyltetrahydrofolate synthetase 1 (MTHFD1), while the mitochondrial compartment is carried out by MTHFD2/2L and methylenetetrahydrofolate dehydrogenase 1-like (MTHFD1L). MTHFD1 is a cytoplasmic trifunctional enzyme with $\mathrm{CH}_{2}$-THF dehydrogenase, 5,10-methenyl-tetrahydrofolate $\left(\mathrm{CH}^{+}-\mathrm{THF}\right)$ cyclohydrolase, and 10-formyl-tetrahydrofolate (10-CHO-THF) synthase activities responsible for cytoplasmic reactions 1, 2, and 3 denoted in Figure 1, respectively. ALDH1L1, a 10-CHO-THF dehydrogenase, is responsible for reaction 4 denoted in Figure 1. MTHFD2/2L is a mitochondrial bifunctional enzyme with $\mathrm{CH}_{2}$-THF dehydrogenase and $\mathrm{CH}^{+}$-THF cyclohydrolase activities responsible for mitochondrial reactions 5 and 6, respectively (Figure 1). Notably, MTHFD2 mainly uses NAD ${ }^{+}$while MTHFD2L mainly uses $\mathrm{NADP}^{+}$to generate NADH and NADPH, respectively. MTHFD1L is a mitochondrial monofunctional enzyme with 10-CHO-THF synthase activity responsible for reaction 7 (Figure 1). ALDH1L2, another 10-CHO-THF dehydrogenase, is responsible for reaction 8. The exchange of THF molecules between the cytoplasmic and mitochondrial compartments is restricted. However, both com- 


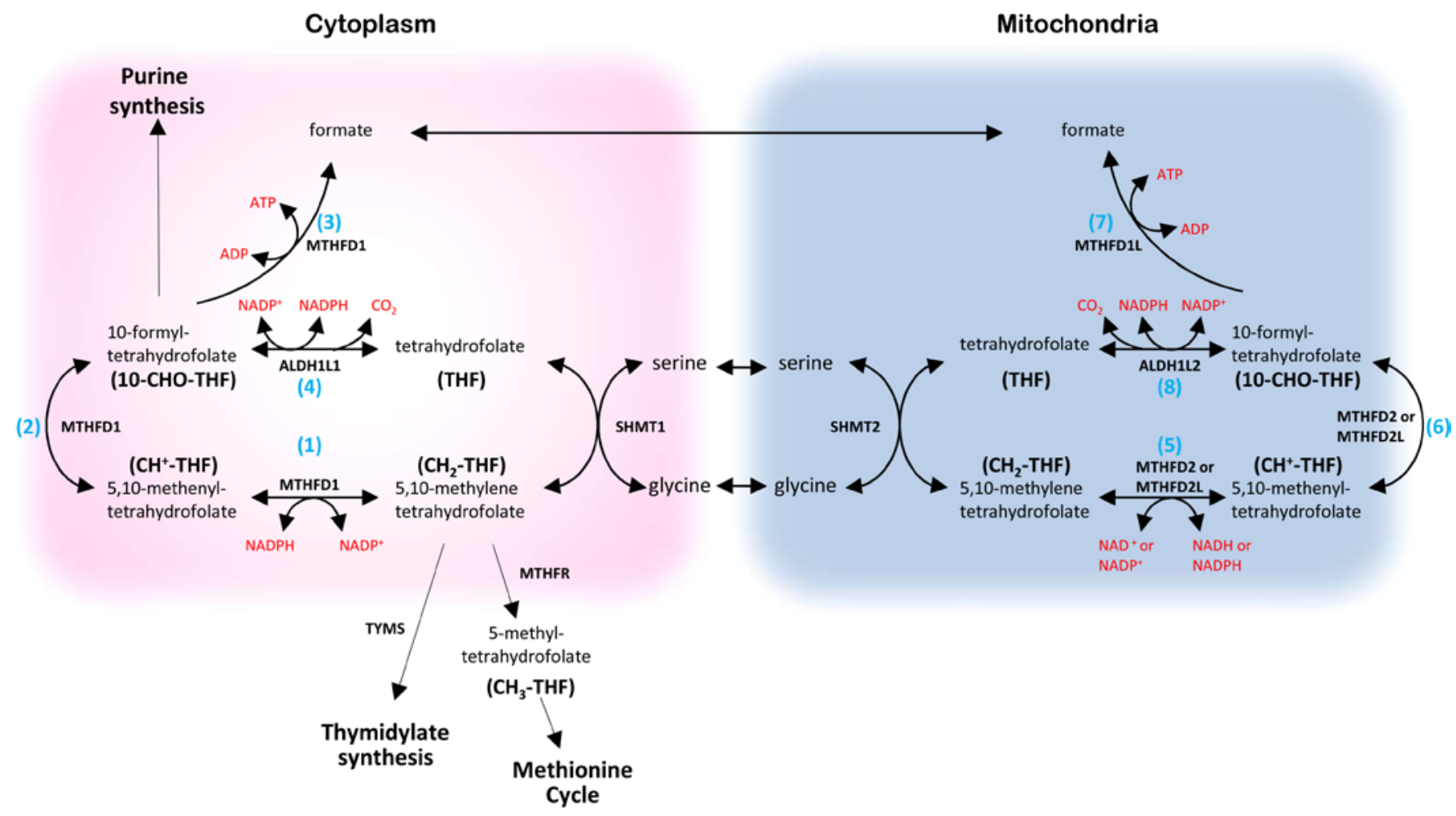

Figure 1. The folate cycle in cancer. Cytosolic and mitochondrial compartments of the folate cycle generate many key metabolites for cell growth.

partments are intimately linked by the transportation of serine, glycine, and formate across the mitochondrial membrane (Figure 1). The continuous cyclical movements in 2 compartments generate many metabolites essential for cell growth. The cytoplasmic folate cycle intermediate 10-CHO-THF is required for purine synthesis, while $\mathrm{CH}_{2}$-THF is required for deoxythymidine monophosphate (pyrimidine) synthesis. $\mathrm{CH}_{2}$-THF is converted to $\mathrm{CH}_{3}-\mathrm{THF}$ by methylenetetrahydrofolate reductase (MTHFR). $\mathrm{CH}_{3}-\mathrm{THF}$ is then connected to the methionine cycle, where the $1 \mathrm{C}$ unit from $\mathrm{CH}_{3}$-THF is donated to homocysteine, generating methionine, the donor of $S$-adenosylmethionine for DNA and histone methylation. Importantly, the folate cycle is an important source of NADPH (8), a major cellular antioxidant. Stable isotope tracing experiments have demonstrated that a majority of $1 \mathrm{C}$ units $(>75 \%)$ entering the cytoplasmic methionine cycle were derived from the mitochondria, further confirming the connection of 2 compartments (9). MTHFD1L is the enzyme catalyzing the last step of the mitochondrial compartment generating formate and could subsequently enter the cytoplasmic compartment. MTHFD1L therefore plays critical roles in folate cycle maintenance (10).

Despite the importance of MTHFD1L in the folate cycle, knowledge about its roles in human diseases, especially cancer, remains largely elusive. Farber made the first observation in 1949 that leukemic cell growth was stimulated by folic acid while folic acid antagonists resulted in temporary remissions in patients with acute leukemia (11). His important discovery on the roles of folate in cancer provided the foundation of the development of chemotherapeutic agents as single or combined treatments for cancer (11). Homozygous Mthfdll-knockout mice exhibited fetal growth delay, neural tube and craniofacial abnormalities, and embryonic lethality (12). In humans, MTHFD1L polymorphisms are closely related to neural tube defects, coronary artery disease, and Alzheimer's disease (13-15). Two microarray gene expression profile studies briefly revealed that MTHFD1L was overexpressed in colorectal and breast cancers $(16,17)$. Discovered in breast cancer cells, miR-9 and miR-197 inhibited MTHFD1L through interacting with its $3^{\prime}$-UTR $(18,19)$. A recent metastudy including microarray data from more than 1,900 tumor tissues of 19 different cancer types suggested that the mitochondrial 1-carbon metabolism is the most deregulated metabolic pathway in human cancers (20). Intriguingly, SHMT2 and MTHFD2 were found to be overexpressed in multiple human cancers and were correlated with shorter survival in breast cancer patients $(16,20,21)$. Knockdown of MTHFD2 in breast cancer cells suppressed proliferation and induced apoptosis (20). Multiple studies indicated that the folate cycle is an important metabolic pathway crucial to cancer growth $(11,20,22-24)$. A recent study showed that the folate cycle, through NADPH production, enabled melanoma cells to endure oxidative stress during metastasis (25).

Hepatocellular carcinoma (HCC), a malignancy transformed from hepatocytes, accounts for $90 \%$ of primary liver cancers. HCC is the fifth most common and second most fatal cancer worldwide. Owing to late symptom presentation, most HCC patients are diagnosed at advanced stages when surgical treatments are deemed unsuitable. Currently, the only FDA-approved targeted therapy for HCC patients, a multikinase inhibitor known as sorafenib, prolongs their lifespan for a mere 3 months $(26,27)$. A better therapeutic regimen is warranted. The liver is a meta- 
A

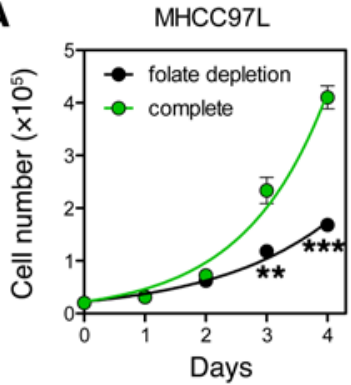

C Transcriptome sequencing, Queen Mary Hospital, HKU

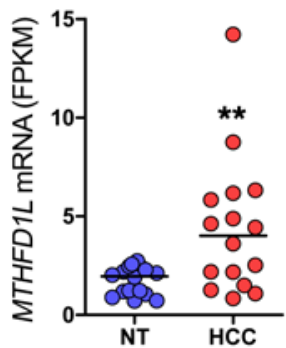

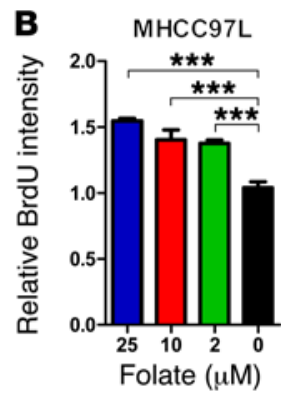

D

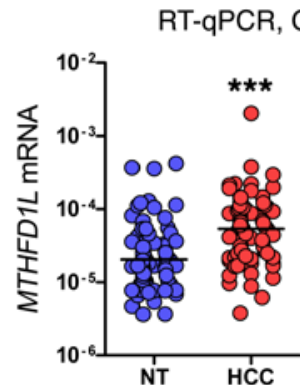

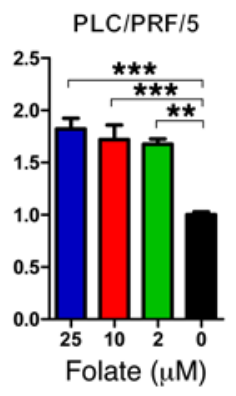

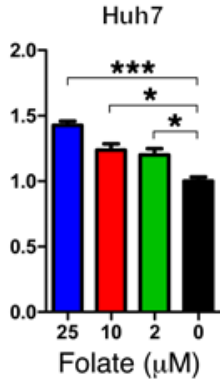

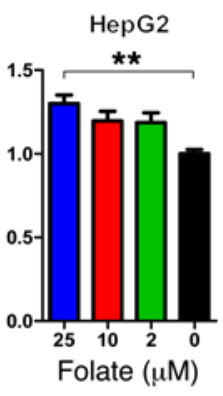

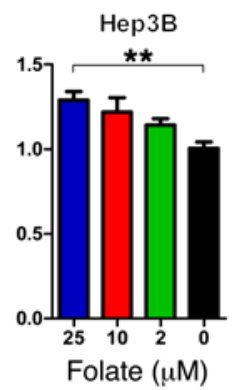

$\mathbf{F}$
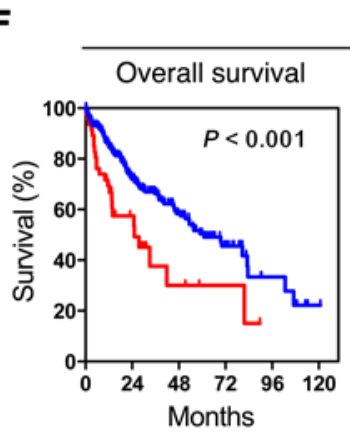

- MTHFD1L High

- MTHFD1L Low
TCGA

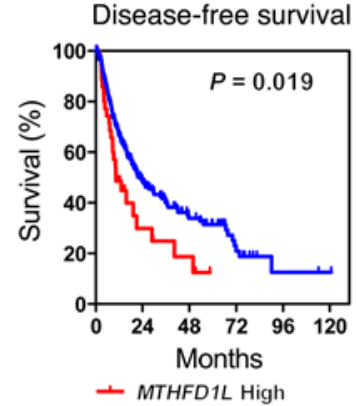

G

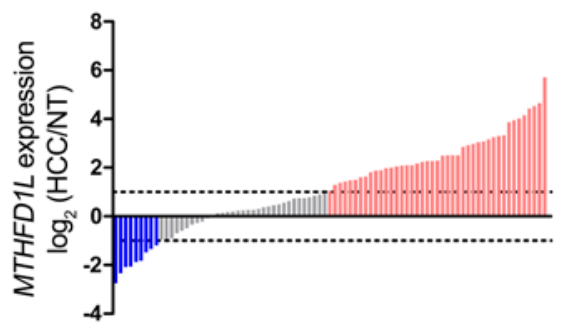

E

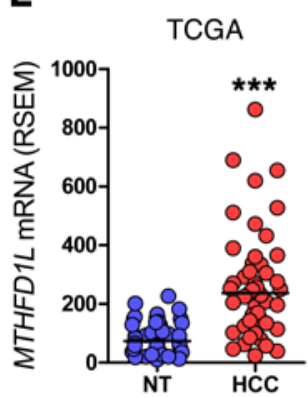

H

- MTHFD1L Low
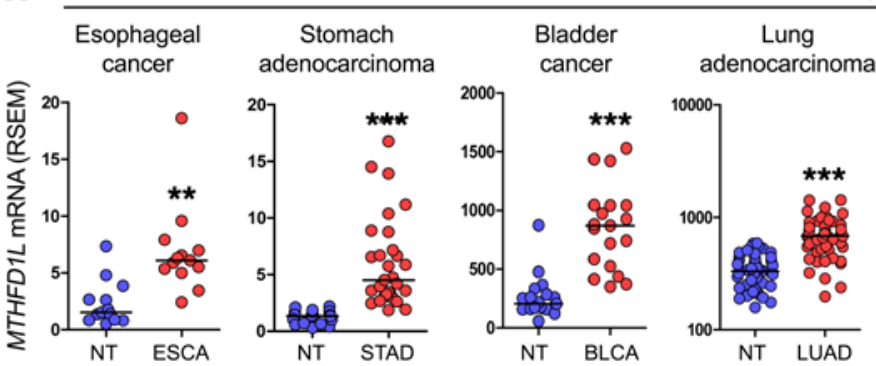

TCGA
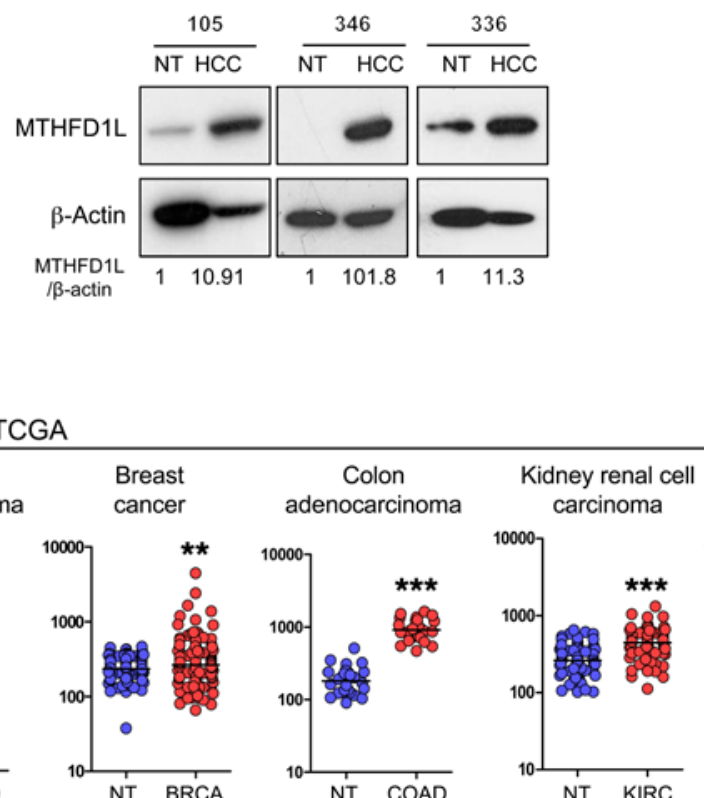

Prostate adenocarcinoma

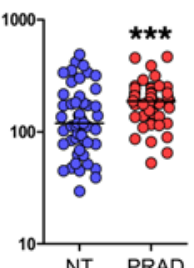

Figure 2. The folate cycle in cancer. (A) An HCC cell line, MHCC97L, was cultured in complete MEM ( $2 \mu \mathrm{M}$ folate) and folate-free MEM, and cell numbers were recorded by cell counter. (B) Different HCC cell lines, MHCC97L, PLC/PRF/5, Huh7, HepG2, and Hep3B, were cultured in MEM containing different concentrations of folate, and BrdU uptake was recorded. (C) MTHFD1L mRNA expression in 16 cases of human HCC and paired nontumorous liver (NT) tissues was detected by transcriptome (RNA) sequencing. FPKM, fragments per kilobase of exon per million fragments mapped; HKU, University of Hong Kong. (D) Left: MTHFD1L mRNA expression in 85 cases of human HCC and paired NT tissues was detected by reverse transcription quantitative PCR (RT-qPCR). Values were calculated based on the following formula: 2-(Ct of MTHFD1L - Ct of 185), where Ct is the cycle threshold. Right: Waterfall plot demonstrated that MTHFD1L overexpression was found in 50.59\% (43/85) of HCC cases. (E) MTHFD1L mRNA expression in 49 human HCC cases from TCGA. RSEM, RNA-Seq expression estimation by expectation maximization. (F) Analysis of TCCA data revealed that HCC patients with high MTHFD1L expression ( $Z$ score $>1$ ) were significantly associated with poorer overall and disease-free survival. (G) MTHFD1L protein expression in 3 representative HCC cases. (H) MTHFD1L mRNA expression in 8 other solid cancers from the TCGA database. A and B: Results are representative of 3 independent experiments. Error bars indicate mean \pm SEM. ${ }^{*} P<0.05,{ }^{* *} P<0.01$, ${ }^{* *} P<0.001$ vs. folate depletion control. A, C, D, and $\mathbf{H}$ : Student's $t$ test. B: 1 -way ANOVA. E: Wilcoxon signed-rank test. F: Kaplan-Meier followed by log-rank test. 
Table 1. Clinicopathological correlation of MTFHD1L upregulation in HCC patients

\begin{tabular}{|c|c|c|c|c|}
\hline & \multicolumn{4}{|c|}{ MTHFD1L expression } \\
\hline & & Normal & Upregulation & $P$ value \\
\hline \multirow[t]{2}{*}{ Gender } & Male & 38 & 26 & 0.290 \\
\hline & Female & 15 & 5 & \\
\hline \multirow[t]{2}{*}{ HBsAg } & Positive & 41 & 26 & 0.775 \\
\hline & Negative & 11 & 5 & \\
\hline \multirow[t]{2}{*}{ Cirrhosis } & Present & 25 & 18 & 0.366 \\
\hline & Absent & 26 & 12 & \\
\hline \multirow[t]{2}{*}{ Tumor size } & $<5 \mathrm{~cm}$ & 21 & 10 & 0.485 \\
\hline & $\geq 5 \mathrm{~cm}$ & 29 & 20 & \\
\hline \multirow[t]{2}{*}{ Venous invasion } & Present & 23 & 22 & $0.020^{A}$ \\
\hline & Absent & 28 & 8 & \\
\hline \multirow[t]{2}{*}{ Tumor microsatellite } & Present & 20 & 20 & $0.037^{A}$ \\
\hline & Absent & 30 & 10 & \\
\hline \multirow[t]{2}{*}{ Direct liver invasion } & Present & 18 & 12 & 0.806 \\
\hline & Absent & 28 & 15 & \\
\hline \multirow[t]{2}{*}{ Tumor encapsulation } & Present & 18 & 8 & 0.464 \\
\hline & Absent & 32 & 22 & \\
\hline \multirow[t]{2}{*}{ Edmonson grading } & $1 / 11$ & 29 & 11 & 0.105 \\
\hline & III/IV & 21 & 19 & \\
\hline \multirow[t]{2}{*}{ pTNM staging } & $1 / I I$ & 25 & 7 & $0.021^{A}$ \\
\hline & III/IV & 26 & 24 & \\
\hline
\end{tabular}

${ }^{A} P<0.05 ; n=85$; categorized based on 4-fold expression. PTNM, pathological tumor node metastasis.

bolic organ responsible for a number of unique metabolic functions, such as blood glucose homeostasis. During HCC development, a series of liver-specific metabolic functions are impaired, accompanied by the acquisition of new metabolic traits that support the infinite growth of HCC cells. Thorough understanding of the metabolic machinery of HCC and its molecular basis is critical to the design of novel diagnostic and therapeutic approaches. In this study, using HCC as a cancer model, we demonstrate the indispensable roles of the folate cycle in cancer progression. Furthermore, we show that a pivotal enzyme of the folate cycle, MTHFD1L, is frequently overexpressed in cancers and confers on cancer cells the ability to survive oxidative stress-induced cell cycle delay and apoptosis. Blockade of the folate cycle by knockdown or knockout of MTHFD1L or by the use of folate analog effectively represses cancer growth and sensitizes cancer cells toward sorafenib treatment.

\section{Results}

MTHFD1L in the folate cycle is significantly overexpressed in human cancers. To understand whether HCC cell proliferation relies on the folate cycle, we cultured an HCC cell line, MHCC97L, in complete medium and folate-depleted medium. Depletion of folate significantly impaired the proliferation rate of MHCC97L (Figure 2A). To confirm that this observation was not cell line-specific, we performed BrdU proliferation assay in MHCC97L as well as 4 additional HCC cell lines, PLC/PRF/5, Huh7, HepG2, and Hep3B. Folate depletion significantly reduced BrdU uptake in different HCC cell lines, further suggesting folate as an important nutrient supporting HCC growth (Figure 2B). To gain insights on the clinical significance of the folate cycle in human HCC, we examined the expression of folate cycle enzymes by transcriptome sequencing in 16 pairs of human HCC and nontumorous liver tissues. Intriguingly, transcriptome sequencing in a small cohort of HCC patients revealed that a critical enzyme connecting the mitochondrial and cytoplasmic compartments of the folate cycle, MTHFD1L, was significantly overexpressed in HCC (Figure 2C). We confirmed MTHFD1L overexpression in an expanded cohort of 85 HCC patients by reverse transcription quantitative PCR (Figure 2D). MTHFD1L overexpression was present in 50.59\% (43/85) of HCC patients by at least 2-fold (Figure 2D). HCC transcriptome sequencing data available in The Cancer Genome Atlas (TCGA) echoed our findings from in-house expression data (Figure 2E). From our in-house HCC samples, we found that MTHFD1L overexpression was closely associated with aggressive clinicopathological features of HCC including the presence of tumor microsatellite formation, venous invasion, and more advanced tumor stages (Table 1). TCGA data revealed that HCC patients with high MTHFD1L expression also have lower overall and disease-free survival rates (Figure 2F). Western blotting further confirmed that MTHFD1L was also overexpressed at the protein level (Figure 2G). Apart from HCC, data retrieved from TCGA also revealed that MTHFD1L overexpression is present in solid cancers including esophageal cancer, stomach adenocarcinoma, bladder cancer, lung adenocarcinoma, breast cancer, colon adenocarcinoma, kidney renal cell carcinoma, and prostate adenocarcinoma (Figure $2 \mathrm{H}$ ).

MTHFD1L is a transcription target of NRF2. Next, we investigated the possible molecular mechanisms driving MTHFD1L overexpression in HCC. A study using lung cancer cells showed that MTHFD2 expression was reduced upon knockdown of nuclear factor (erythroid-derived 2)-like 2 (NRF2), a transcription factor that acts as the central regulator for redox homeostasis (28). We speculated that MTHFD1L is also a transcriptional target of NRF2. NRF2 expression is tightly regulated by kelch-like ECHassociated protein 1 (KEAP1), a negative regulator that targets NRF2 for proteosomal degradation (29). Oxidative stress prevents KEAP1-mediated degradation of NRF2, allowing the translocation of stabilized NRF2 into the nucleus, thereby binding to genes encompassing antioxidant response elements (AREs) 5'-TMAnnRTGAYnnnGCRwwww-3' ( $\mathrm{M}=\mathrm{A} / \mathrm{C}, \mathrm{R}=\mathrm{A} / \mathrm{G}, \mathrm{Y}=\mathrm{C} / \mathrm{T}$, $\mathrm{w}=\mathrm{A} / \mathrm{T}$, and $\mathrm{n}=$ random bases) (29). NRF2 initiates transcription of genes that counteract oxidative stress. We found 3 putative AREs in MTHFD1L and confirmed that NRF2 bound to these AREs by ChIP assay in a human HCC cell line, MHCC97L (Figure 3A). We generated NRF2-overexpressing MHCC97L cells by CRISPR-dCas9 Synergistic Activation Mediator (SAM) system and found that MTHFD1L mRNA expression was significantly induced (Figure 3B). Reversely, MTHFD1L mRNA and protein expressions were reduced upon knockdown of NRF2 in different HCC cell lines, MHCC97L, PLC/PRF/5, and Huh7 (Figure 3, C and D). These data converged to show that MTHFD1L is transcriptionally regulated by NRF2.

Knockdown of MTHFD1L impedes HCC proliferation through induction of oxidative stress and ROS-associated cell cycle delay. To understand the functions of MTHFD1L, we generated MTHFD1L-overexpressing MHCC97L cells using CRISPR- 
A
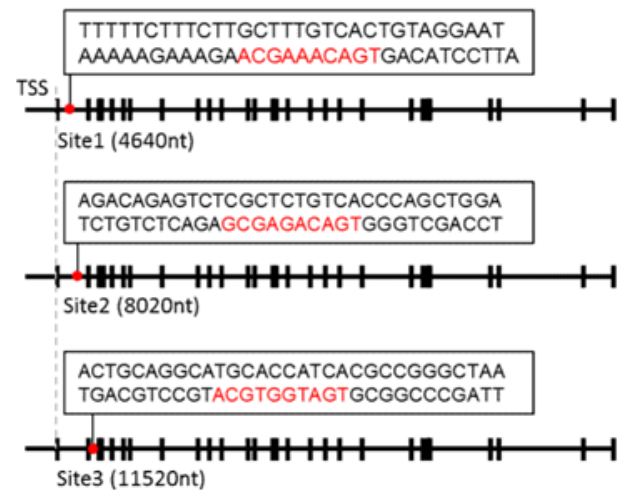

ML-Site1
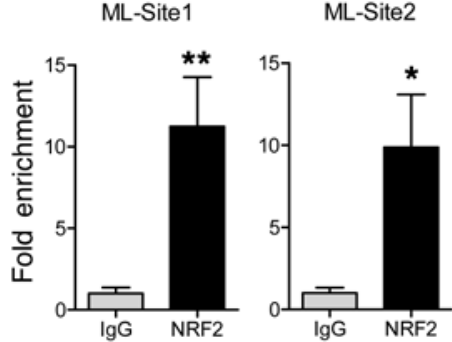

ML-Site3

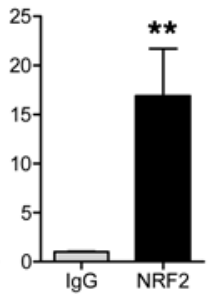

B

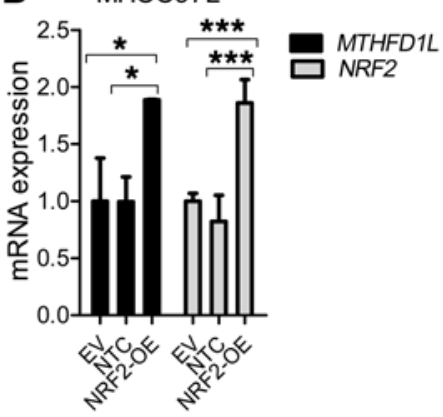

D $\quad$ MHCC97L PLC/PRF/5 Huh7

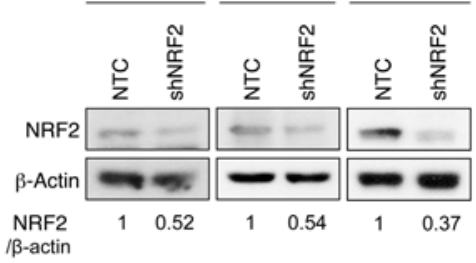

C PLC/PRF/5

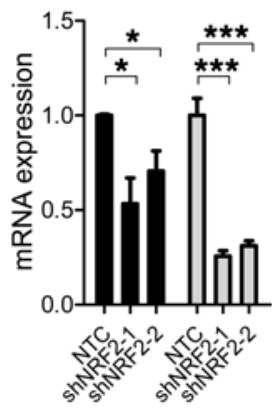

MHCC97L PLC/PRF/5 Huh7

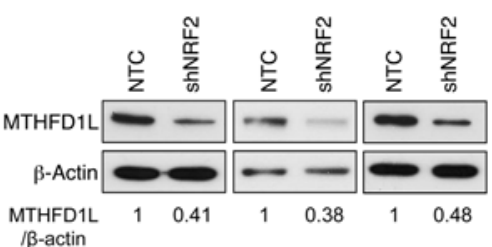

Figure 3. Regulation of MTHFD1L. (A) Top: Three AREs are located in MTHFD1L. Bottom: ChIP assay using NRF2 and IgC antibodies in MHCC97L cells confirmed that NRF2 bound to the putative AREs of MTHFD1L. (B) NRF2-overexpressing (OE) HCC cells were established by CRISPR-dCas9 SAM system. MTHFD1L and NRF2 mRNA expressions in MHCC97L-empty vector (EV), MHCC97L-nontarget control (NTC), and NRF2-OE HCC cells were detected by RT-qPCR and normalized to 18S. (C) NRF2-knockdown HCC cells were established by an shRNA approach. MTHFD1L and NRF2 mRNA expressions in PLC/PRF/5-NTC and PLC/PRF/5shNRF2 cells were quantitated by RT-qPCR and normalized to 18S. (D) NRF2 and MTHFD1L protein expressions in various NRF2-knockdown (MHCC97L-NTC, -shNRF2; PLC/PRF/5-NTC, -shNRF2; Huh7-NTC, -shNRF2) HCC cells. Band intensities were quantitated by ImageJ (NIH) and normalized to $\beta$-actin. A-C: Results are representative of 3 independent experiments. Error bars indicate mean \pm SEM. ${ }^{*} P<0.05,{ }^{* *} P<0.01,{ }^{* * *} P<0.001$ vs. NTC or EV. Student's $t$ test.

dCas9 SAM system (Figure 4A). As a result of MTHFD1L overexpression, we found that MTHFD1L increased the intracellular $\mathrm{NADPH} / \mathrm{NADP}^{+}$ratio and reduced intracellular ROS level (Figure 4, B and C). Reversely, we also generated MTHFD1L-knockdown MHCC97L cells (Figure 4D) and found that knockdown of MTHFD1L reduced the intracellular NADPH/NADP ${ }^{+}$ratio, leading to the accumulation of ROS (Figure 4, E and F). Knockout of MTHFD1L by CRISPR-Cas9 gene editing system in MHCC97L and $\mathrm{PLC} / \mathrm{PRF} / 5$ cells consistently reduced the intracellular $\mathrm{NADPH} / \mathrm{NADP}^{+}$ratio and increased intracellular ROS level (Figure $4, \mathrm{G}-\mathrm{L}$ ). ROS induces DNA damage by incorporating into the DNA of the cells to form 8-hydroxy-2-deoxyguanosine $(8-\mathrm{OH}-$ $\mathrm{dG})$. We found that DNA of the MTHFD1L-knockdown and -knockout cells contained a higher level of 8-OH-dG (Figure 5A and Supplemental Figure 1A; supplemental material available online with this article; https://doi.org/10.1172/JCI90253DS1). Next, we evaluated the mitochondrial functions of MTHFD1Lknockdown cells by the XFp Cell Mito Stress Test, in which oligomycin, FCCP, and antimycin A and rotenone were sequentially added to the cells. As these drugs target different parts of the electron transport chain, oxygen levels detected at different drug injection time points generate a mitochondrial respiration profile that reflects the mitochondrial activity (Figure 5B). Noticeable reductions of basal respiration (oxygen consumption) and respiratory chain capacity as a result of MTHFD1L knockdown indicated the importance of MTHFD1L in maintaining proper mitochondrial function (Figure 5B). We further asked whether the metabolic vulnerabilities created by MTHFD1L knockdown would affect the proliferative output of HCC cells in vitro. As anticipated, knockdown and knockout of MTHFD1L significantly repressed proliferation of MHCC97L cells (Figure 5C and Supplemental Figure 1B). To confirm that our observation was not cell line-specific, we generated MTHFD1L-knockdown clones in 3 additional HCC cell lines, PLC/PRF/5, HepG2, and Hep3B (Supplemental Figure 2). Consistently, we found that knockdown of MTHFD1L caused ROS accumulation and repressed HCC proliferation in multiple HCC cell lines. We proceeded to ask whether ROS accumulation caused by MTHFD1L knockdown affects the cell cycle profile of HCC cells. HCC cells were first synchronized with nocodazole in the G2/M phase (Supplemental Figure 3). Cells were then released from nocodazole treatment. Propidium iodide staining showed that a greater percentage of MTHFD1L-knockdown and -knockout cells remained in the G1 phase relative to nontarget control (NTC) cells (Figure 5, D and E; Supplemental Figure 1C; and Table 2). Administration of different antioxidants, reduced glutathione (GSH and GSH-EE) and $\mathrm{N}$-acetylcysteine (NAC), allowed the reentry of MTHFD1Lknockdown cells into the $\mathrm{S}$ and $\mathrm{G} 2 / \mathrm{M}$ phases (Figure 5, D and E). MTHFD1L-mediated cell cycle delay was thus confirmed to be caused by ROS accumulation. We further confirmed that GSH could reduce ROS accumulation in MTHFD1L-knockdown cells (Figure 5F). BrdU proliferation assay further showed that GSH could partially rescue the proliferative deficiency of MTHFD1Lknockdown HCC cells (Figure 5G). 

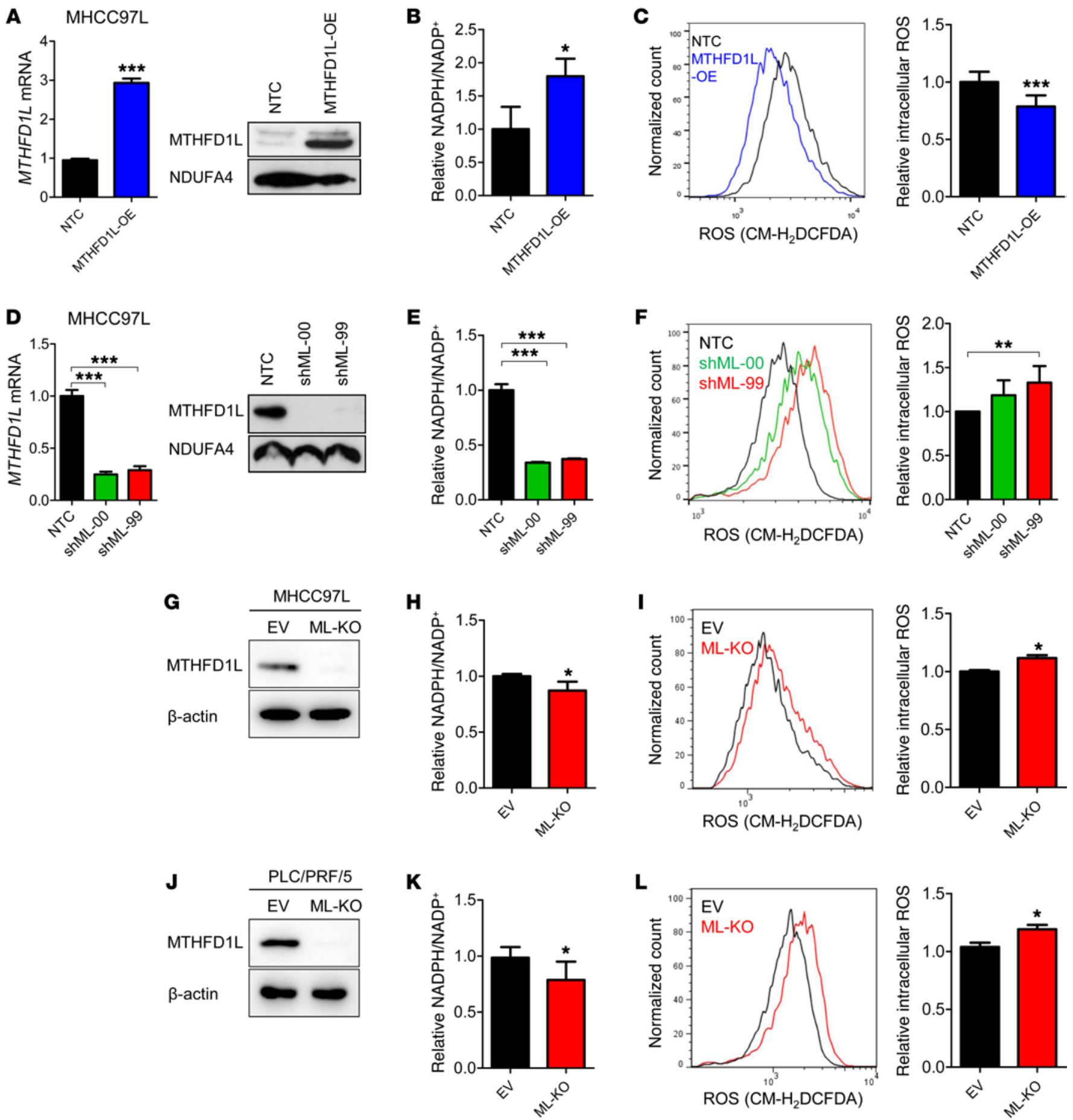

Figure 4. MTHFD1L increased NADPH/NADP+ levels and reduced ROS. (A) MTHFD1L-overexpressing (OE) HCC cells were established by CRISPR-dCas9 SAM system. mRNA and protein expressions of MTHFD1L in MHCC97L-MTHFD1L-OE stable cells that express single guide RNA (sgRNA) targeting MTHFD1L and MHCC97L-NTC stable cells that express nontarget control (NTC) sgRNA. (B) Levels of NADPH/NADP+ in MHCC97L-NTC and -MTHFD1LOE cells. (C) Levels of intracellular ROS in MHCC97L-NTC and -MTHFD1L-OE cells. (D) MTHFD1L-knockdown HCC cells were established by shRNA approach. mRNA and protein expressions of MTHFD1L in MHCC97L-NTC, -shMTHFD1L-00 (shML-00), and -shMTHFD1L-99 (shML-99) stable cells. (E) Levels of NADPH/NADP+ in MHCC97L-NTC, -shML-00, and -shML-99 stable cells. (F) Levels of intracellular R0S in MHCC97L-NTC, -shML-00, and -shML-99 stable cells. (G) MTHFD1L-knockout (KO) MHCC97L cells were established by CRISPR-Cas9 system. MTHFD1L protein expression in MHCC97L stable cells expressing empty vector control (EV) and sgRNA against MTHFD1L (ML-KO). (H) Levels of NADPH/NADP+ in MHCC97L-EV and -MTHFD1LKO (ML-KO) stable cells. (I) Levels of intracellular ROS in MHCC97L-EV and -ML-KO cells. (J) MTHFD1L-knockout (KO) PLC/PRF/5 cells were established by CRISPR-Cas9 system. MTHFD1L protein expression in PLC/PRF/5 stable cells expressing empty vector control (EV) and sgRNA against MTHFD1L (ML-KO). (K) Levels of NADPH/NADP+ in PLC/PRF/5-EV and MTHFD1L-KO (ML-KO) stable cells. (L) Levels of intracellular ROS in PLC/PRF/5-EV and $-\mathrm{ML}-\mathrm{KO}$ cells. Results are representative of 3 independent experiments. Error bars indicate mean $\pm \mathrm{SEM} .{ }^{*} P<0.05,{ }^{* *} P<0.01,{ }^{* * *} P<0.001 \mathrm{vs}$. NTC or EV. A-C, H, I, K, and L: Student's $t$ test. D-F: 1-way ANOVA. 

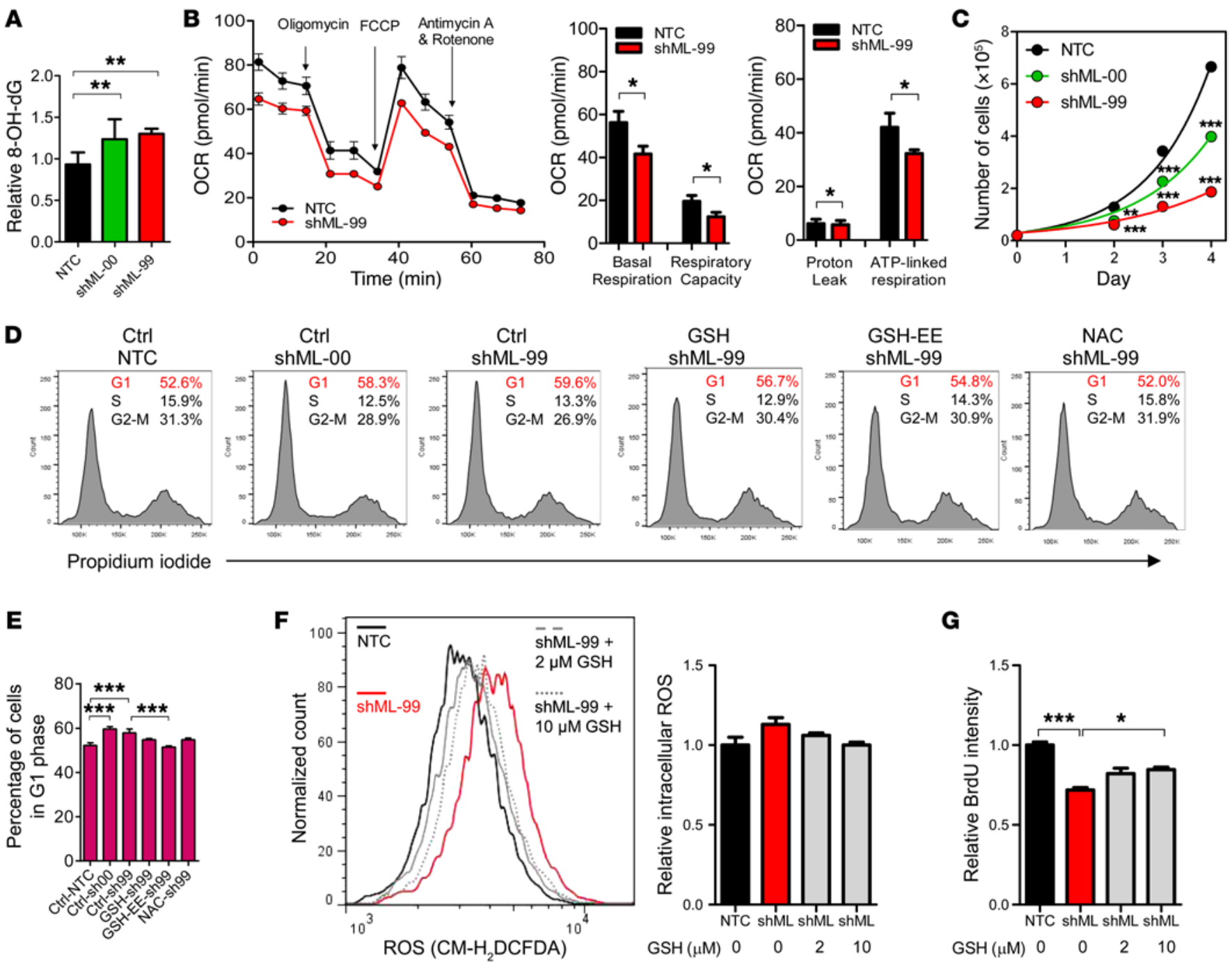

Figure 5. Knockdown of MTHFD1L caused cell cycle delay. (A) 8-OH-dG levels in MHCC97L-NTC, -shML-00, and -shML-99 cells. (B) Oxygen consumption rate (OCR) in MHCC97L-NTC and -shML-99 cells was determined by XFp Analyzer (Seahorse Bioscience). Basal respiration, respiratory capacity, proton leak, and ATP-linked respiration were calculated based on the OCRs at different points when oligomycin, FCCP, and antimycin A and rotenone were sequentially added to cells. (C) Cell proliferation rate of MHCC97L-NTC, -shML-00, and-shML-99. An equal number of cells was seeded on day 0. Cell numbers were recorded daily by cell counting. (D) Representative pictures of flow cytometry analyses of propidium iodide staining in MHCC97L-NTC, -shML-00, and -shML-99 stable cells that were synchronized by $200 \mathrm{ng} / \mathrm{ml}$ nocodazole for 16 hours and released for 9 hours in the presence of vehicle control and antioxidants $10 \mu \mathrm{M} \mathrm{GSH}, 0.1 \mathrm{mM}$ CSH-EE, and $2 \mathrm{mM}$ NAC. (E) Percentage of cells in G1 phase. (F and $\mathbf{G}$ ) Levels of intracellular ROS (F) and BrdU uptake (G) in MHCC97L-NTC and -shML-99 cells treated with vehicle or different concentrations of reduced GSH. Results are representative of 3 independent experiments. Error bars indicate mean \pm SEM. ${ }^{*} P<0.05,{ }^{* *} P<0.01,{ }^{* *} P<0.001$ vs. NTC, or as indicated. A, C, and E-G: 1 -way ANOVA. B: Student's $t$ test.

Knockdown of MTHFD1L alters the metabolic program of HCC cells. To perceive the metabolic impacts of MTHFD1L on cancer cells, metabolomic profiling using capillary electrophoresis time-of-flight mass spectrometry (CE-TOFMS) was performed on MHCC97L-NTC and -shMTHFD1L cells (Supplemental Figure 4). Knockdown of MTHFD1L greatly altered the metabolic profiles of HCC cells (Supplemental Figure 4A). Principal component analysis demonstrated that the extractions were consistent in the triplicates of each group (Supplemental Figure 4B). Strikingly, the knockdown of MTHFD1L caused accumulations of glyceraldehyde 3-phosphate (G3P), 3-phosphoserine (3PSer), and serine. As a serine molecule is required to initiate the folate cycle (Figure 6A), MTHFD1L knockdown resulted in the truncation of the folate cycle, thus blocking the transfer of a carbon unit from serine to THF, ultimately causing an accumulation of serine and 3PSer (Figure 6B).

Interestingly, the glycolytic intermediates glucose 1-phosphate, glucose 6-phosphate, fructose 6-phosphate, fructose 1,6-bisphosphate, pyruvate, and lactate accumulated in MTHFD1L-knockdown HCC cells, suggesting a coordination between the folate cycle and glycolysis (Figure 6B). Another notably interesting pattern of metabolic changes in the TCA cycle was observed in the MTHFD1L-knockdown HCC cells (Figure 6C). We found that the levels of TCA cycle metabolic intermediates derived from pyruvate/acetyl-CoA, including citrate, cis-aconitate, and isocitrate, decreased (Figure 6D). Meanwhile, the levels of TCA 
Table 2. $P$ values between groups (percentage of cells in G1 phase)

\begin{tabular}{|c|c|c|c|c|c|c|}
\hline & Ctrl-NTC & Ctrl-sh00 & Ctrl-sh99 & GSH-sh99 & GSH-EE-sh99 & NAC-sh99 \\
\hline Ctrl-NTC & - & $<0.0001$ & 0.0006 & 0.2464 & 1.0000 & 0.2464 \\
\hline Ctrl-sh00 & - & - & 1.0000 & 0.0026 & $<0.0001$ & 0.0026 \\
\hline Ctrl-sh99 & - & - & - & 0.0712 & 0.0002 & 0.0712 \\
\hline GSH-sh99 & - & - & - & - & 0.0473 & 1.0000 \\
\hline GSH-EE-sh99 & - & - & - & - & - & 0.0473 \\
\hline NAC-sh99 & - & - & - & - & - & - \\
\hline
\end{tabular}

cycle metabolic intermediates that could be derived from glutamine, including $\alpha$-ketoglutarate, succinate, fumarate, malate, and oxaloacetate, increased (Figure 6D). This suggested that glutamine might replenish the TCA cycle to compensate for the effects of folate cycle defect. Unexpectedly, levels of nucleotides as well as methionine and its derivative $S$-adenosylmethionine were not decreased upon knockdown of MTHFD1L (Supplemental Figure 5 , A and B). NADPH is required for lipid synthesis, which supports HCC development (30-32). We performed Oil Red O staining to quantitate the levels of triglycerides and lipids in the MTHFD1Lknockdown HCC cells and in HCC tumors treated with antifolate drug (methotrexate, MTX); however, no significant difference in lipid metabolism could be detected (Supplemental Figure 5, C and D). Our data suggested that the folate cycle is mainly used for oxidative stress defense in HCC cells.

Inhibition of the folate cycle sensitizes HCC cells to sorafenib treatment in vitro. Sorafenib is the only FDA-approved drug for HCC patients. Our next question was whether the inhibition of the folate cycle would sensitize HCC cells to sorafenib treatment. Sorafenib almost completely retarded the proliferation of MTHFD1L-knockdown HCC cells (Figure 7A). Furthermore, oxidative stress was further elevated by sorafenib in the MTHFD1L-knockdown HCC cells (Figure 7B). More strikingly, sorafenib increased the percentage of apoptotic cells in the MTHFD1L-knockdown HCC cells (Figure 7C). To achieve folate cycle suppression through pharmacological inhibition, the effects of MTX in HCC were investigated. We found that MTX was able to act synergistically with sorafenib to suppress HCC cell proliferation in vitro (Figure 7D). Oxidative stress was also profoundly induced by sorafenib in MTX-treated cells (Figure 7E). Consistent with the knockdown of MTHFD1L, sorafenib significantly elevated apoptosis in HCC cells that were treated with MTX (Figure 7F).

Inhibition of the folate cycle represses HCC growth and sensitizes HCC cellsto sorafenibtreatment invivo. To examine whether the metabolic defects elicited by MTHFD1L knockdown inhibit HCC progression in vivo, we performed orthotopic liver implantations with MHCC97L-NTC and -shMTHFD1L cells. Six weeks after implantation, not only did we find that knockdown of MTHFD1L in HCC cells profoundly suppressed the growth of primary tumors (Figure $8 \mathrm{~A}$ ); the incidence of lung metastasis was noticeably reduced (Figure $8 \mathrm{~B}$ ). Ex vivo imaging showed that lung metastasis was found in 0 of 6 mice and 1 of 6 mice in the 2 MTHFD1L-knockdown groups as opposed to its occurrence in 5 of 6 mice from the NTC group (Figure $8 \mathrm{~B}$ ). Histological analysis suggested that tumors derived from MHCC97L-NTC cells were more aggressive than those derived from MHCC97L-shMTHFD1L cells. Many of the tumors derived from the inoculation of MTHFD1L-knockdown cells were encapsulated and regular, while all tumors derived from control HCC cell inoculation exhibited invasive tumor growth fronts (Figure $8 \mathrm{C}$ and Table 3). Furthermore, many necrotic regions could be found in the tumors derived from the MTHFD1L-knockdown cells, suggesting that MTHFD1L-knockdown HCC cells could not survive as well as the control HCC cells. In line with the in vitro data, we found that HCCs with knockdown of MTHFD1L were more sensitive to sorafenib in a subcutaneous tumor model (Figure 8, D-F). Next, we investigated whether pharmacological inhibition of the folate cycle by MTX would sensitize HCC cells to sorafenib treatment. MTX worked synergistically with sorafenib to suppress the growth of subcutaneous HCC tumors derived from MHCC97L as well as patient-derived tumor xenografts in nude mice (Figure 9, A-F). To further confirm our observation in immune-competent mice, we orthotopically implanted a mouse HCC cell line (Hepa 1-6) derived from C57BL/6 mouse background into the livers of C57BL/6 mice. Consistently, we observed synergism between MTX and sorafenib (Figure 9, G and $\mathrm{H}$ ). MTX and sorafenib treatments are well tolerated in mice (Supplemental Figure 6), highlighting the translational value of this new therapeutic regimen for HCC patients.

\section{Discussion}

Over the past decade, research efforts have been devoted to understanding the metabolic advantages of the Warburg effect. Extensive studies focused on particularly the molecular alterations that drive the metabolism of glucose, the major nutrient and participant of the Warburg effect. On the other hand, folate, a major precursor for the folate cycle belonging to the 1-carbon metabolism, received very little attention. The folate cycle is critical for maintenance of various biological events, such as epigenetic modifications, nucleotide synthesis, and antioxidant productions. Driven by our initial observation of growth impairment in folate-depleted HCC cells, we sought to understand the regulation and roles of the folate cycle in HCC development. In this study, we first demonstrated that the major function of the folate cycle in HCC cells is redox maintenance. We demonstrated that a crucial folate cycle component, MTHFD1L, was frequently upregulated in human cancers under the control of NRF2. Knockdown or knockout of MTHFD1L effectively reduced NADPH level and caused ROS accumulation and ROS-associated cell cycle delay, as well as HCC growth hindrance in vitro and in vivo. 
A

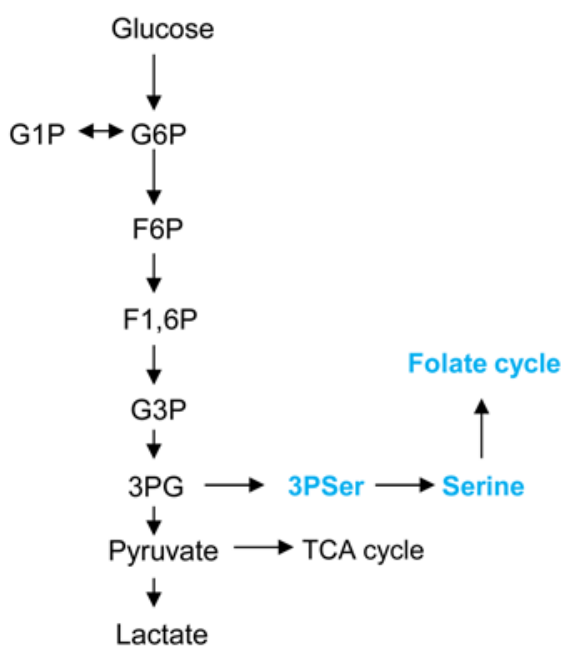

B
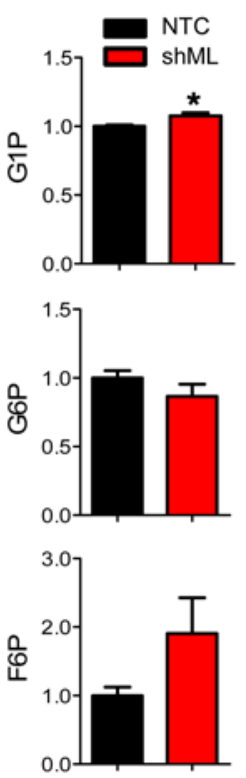
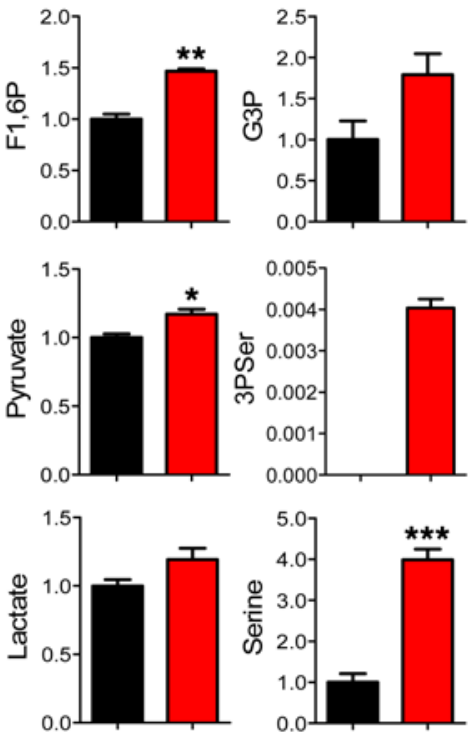

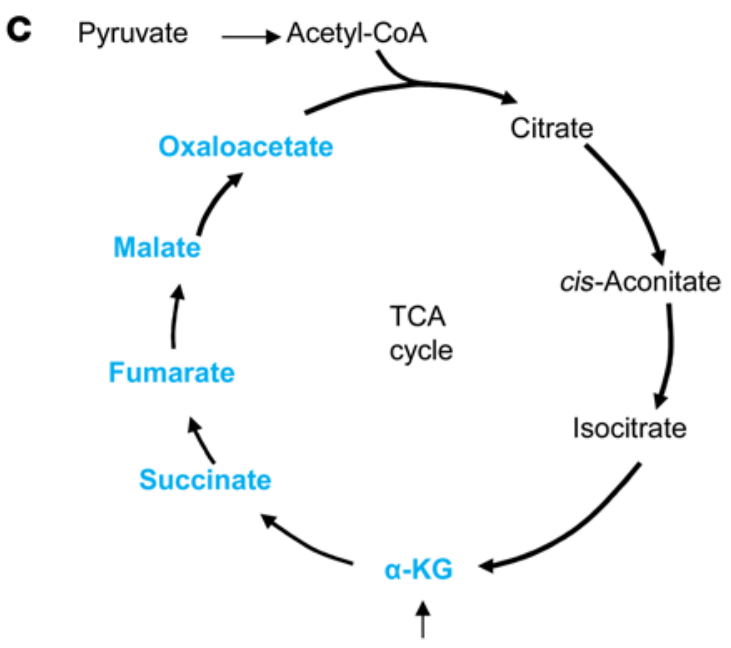

D

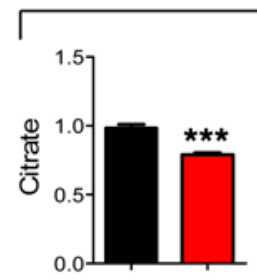

TCA cycle
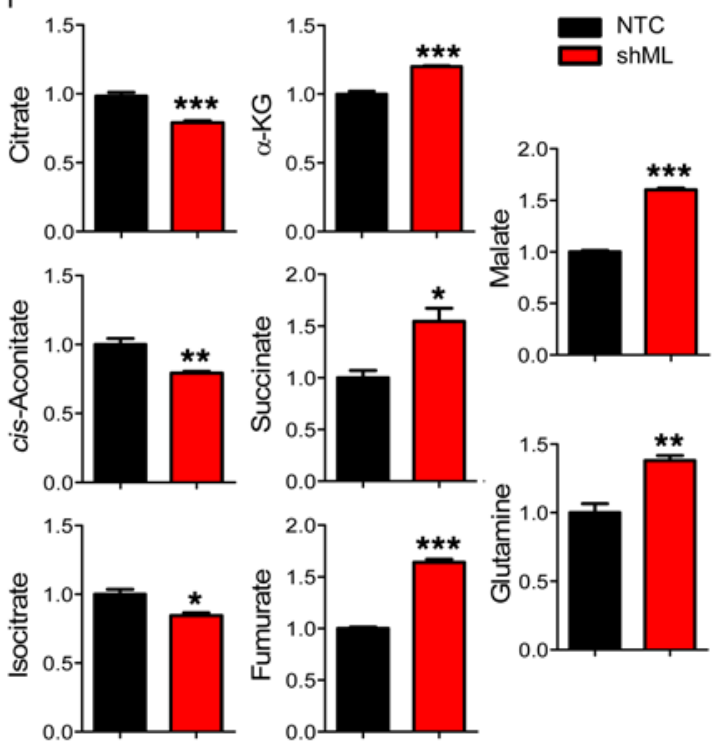

Figure 6. Knockdown of MTHFD1L caused serine accumulation. (A) Metabolic intermediates and reactions in glycolysis. (B) Quantification of metabolic intermediates in glycolysis and serine metabolism by CE-TOFMS. (C) Metabolic intermediates and reactions in TCA cycle. (D) Quantification of metabolic intermediates in TCA cycle by CE-TOFMS. G6P, glucose 6-phosphate; F6P, fructose 6-phosphate; F1,6P, fructose 1,6-bisphosphate; C3P, glyceraldehyde 3-phosphate; 3PG, 3-phosphoglycerate; 3PSer, 3-phosphoserine; $\alpha$-KG, $\alpha$-ketoglutarate. Values of all metabolites were normalized to NTC unless indicated. For 3PSer, absolute values from CE-TOFMS are shown in the graph, as no 3PSer could be detected in the NTC group, so values could not be normalized. Results are representative of 3 independent experiments. Error bars indicate mean $\pm \mathrm{SEM} .{ }^{*} P<0.05,{ }^{* *} P<0.01,{ }^{* *} P<0.001 \mathrm{vs}$. NTC. Student's $t$ test.

NADPH serves as an antioxidant molecule by maintaining glutathione and thioredoxin in reduced states, allowing donation of their electrons to stabilize ROS. NADPH is produced by 4 groups of enzymes: (a) glucose 6-phosphate dehydrogenase (G6PD), (b) malic enzymes, (c) isocitrate dehydrogenases (IDHs), and (d) MTHFDs. The first 3 groups of enzymes are tightly linked with glucose metabolism, and their involvements in cancer development are well documented. However, little information could be found for MTHFDs. G6PD, a key enzyme in the pentose phos- phate pathway (PPP), was overexpressed in HCC owing to the inactivation of PTEN (33). Malic enzymes were overexpressed in human HCC (34) and were found to be repressed by p53 in osteosarcoma cells (35). IDH mutations are frequent in cancers, and IDH mutants have altered enzymatic activities causing the conversion of $\alpha$-ketoglutarate to an oncometabolite, 2-hydroxyglutarate (36), leading to aberrant histone and DNA methylations in the cancer genome (37). Notably, all IDH mutations are heterozygous $(38,39)$, meaning the NADPH-producing ability of wild-type IDH 

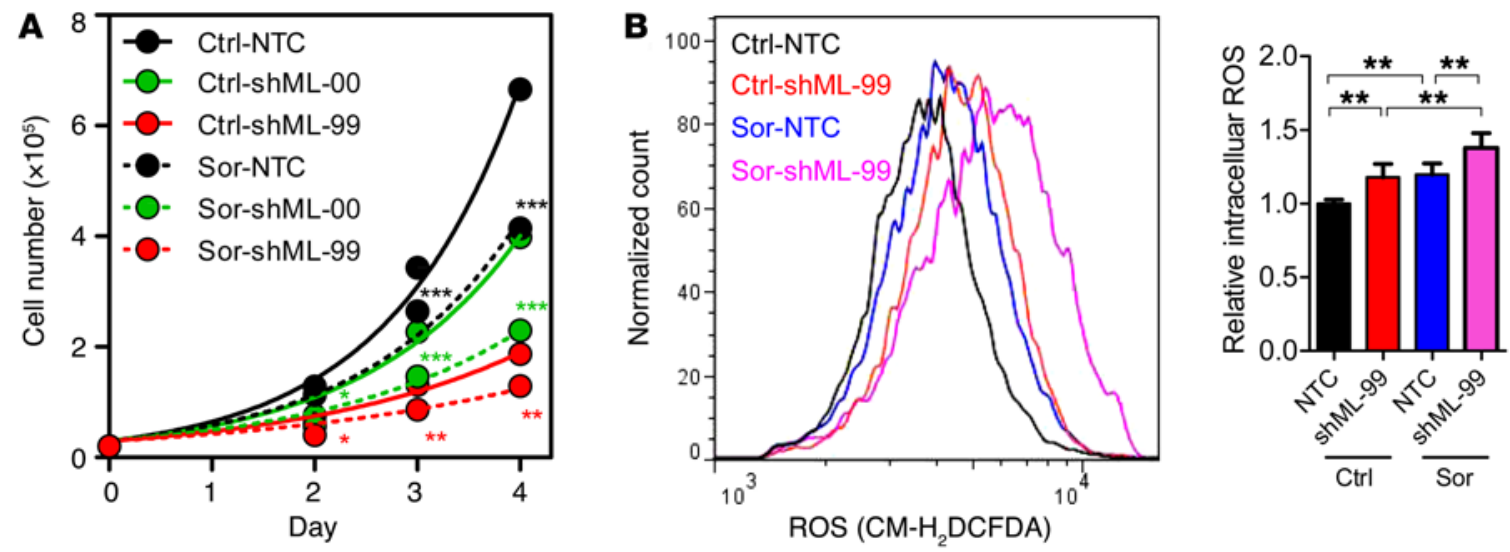

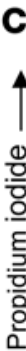
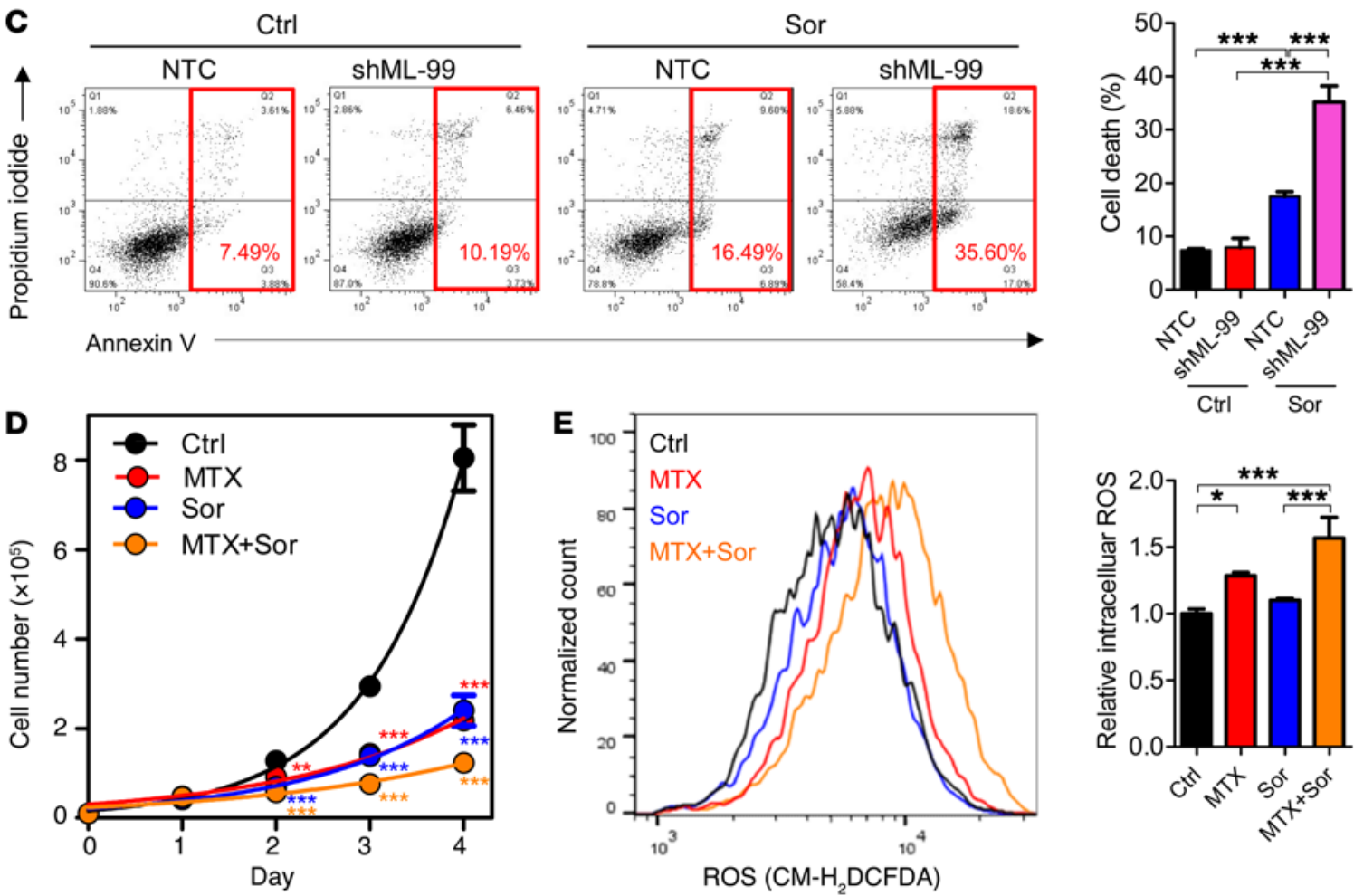

F
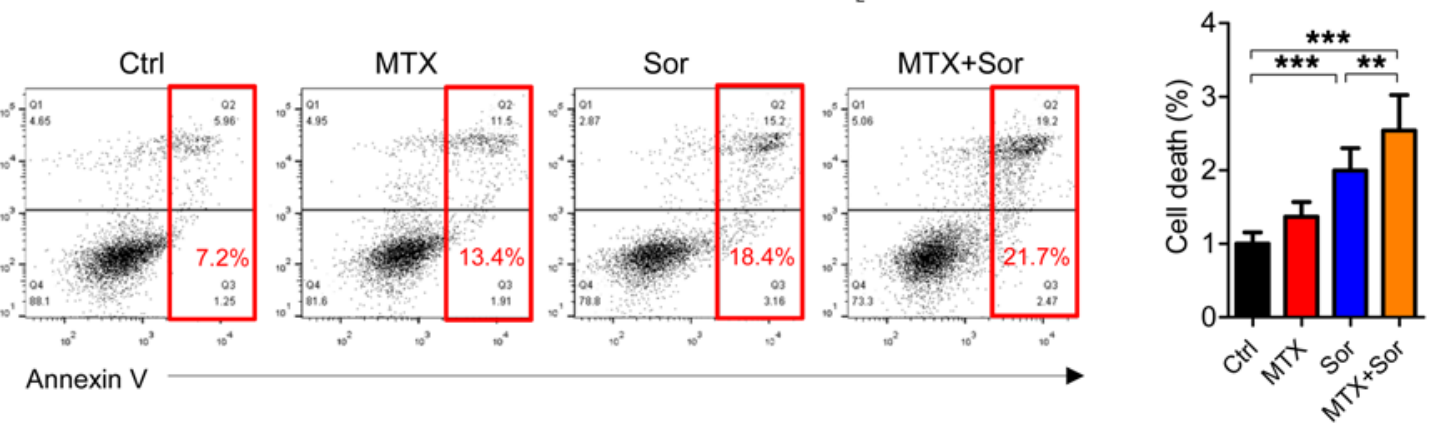

Figure 7. Genetic knockdown of MTHFD1L and pharmacological inhibition of the folate cycle sensitized HCC cells to sorafenib treatment via enhancing oxidative stress. (A) Proliferation of MHCC97L-NTC, -shML-00, and -shML-99 cells in the presence of vehicle control (Ctrl) and $2.5 \mu \mathrm{M}$ sorafenib (Sor). (B) Representative flow cytometry analysis (left) and quantification (right) of ROS (CM-H DCFDA) staining in MHCC97L-NTC and -shML-99 cells treated with vehicle control or $4 \mu \mathrm{M}$ sorafenib for 24 hours. (C) Representative flow cytometry analysis (left) and quantification (right) of annexin $V$ and propidium iodide staining in MHCC97L-NTC and -shML-99 cells treated with vehicle control or $4 \mu \mathrm{M}$ sorafenib for 24 hours. (D) Proliferation of MHCC97L cells treated with vehicle control, $0.01 \mu \mathrm{M}$ MTX alone, $4 \mu \mathrm{M}$ sorafenib alone, and $0.01 \mu \mathrm{M}$ MTX and $4 \mu \mathrm{M}$ sorafenib (MTX+Sor). (E) Representative flow cytometry analysis (left) and quantification (right) of ROS (CM-H DCFDA) staining in MHCC97L cells treated with vehicle control, $0.01 \mu \mathrm{M}$ MTX alone, $4 \mu \mathrm{M}$ sorafenib alone, and $0.01 \mu \mathrm{M}$ MTX and $4 \mu \mathrm{M}$ sorafenib (MTX+Sor) for 24 hours. (F) Representative flow cytometry analysis (left) and quantification (right) of annexin $V$ and propidium iodide staining in MHCC97L cells treated with vehicle control, $0.01 \mu \mathrm{M}$ MTX alone, $4 \mu \mathrm{M}$ sorafenib alone, and $0.01 \mu \mathrm{M}$ MTX and $4 \mu \mathrm{M}$ sorafenib (MTX+Sor) for 22 hours. Results are representative of 3 independent experiments. Error bars indicate mean $\pm \mathrm{SEM} .{ }^{*} P<0.05,{ }^{* *} P<0.01,{ }^{* * *} P<0.001$ vs. Ctrl, Ctrl-NTC, or as indicated. 1-way ANOVA. 
A

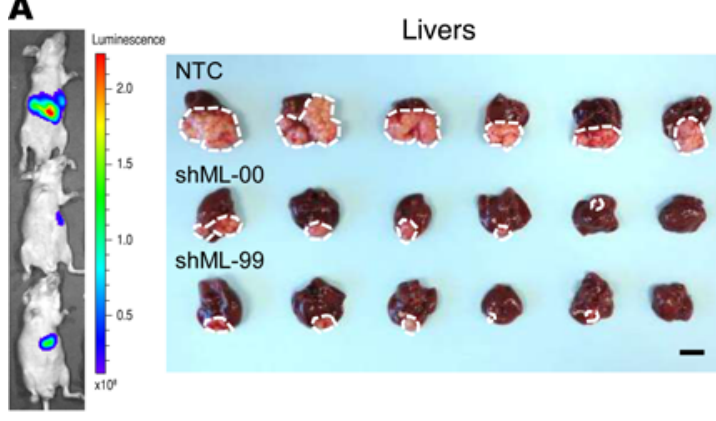

c

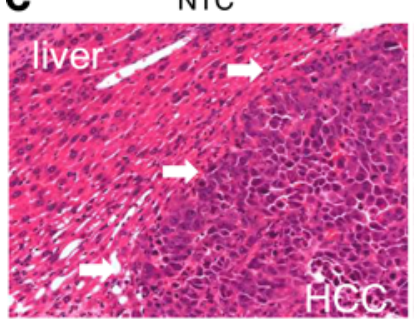

E

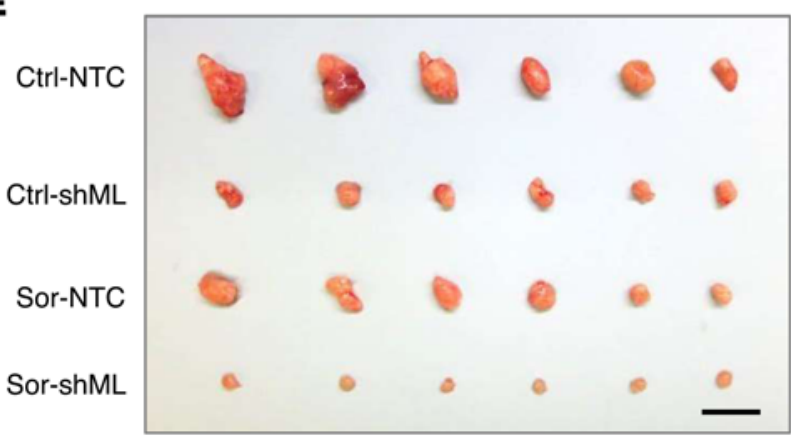

shML-00

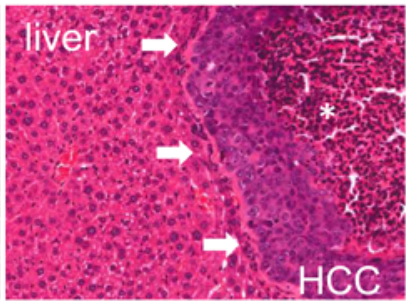

B

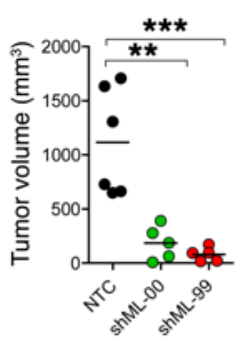

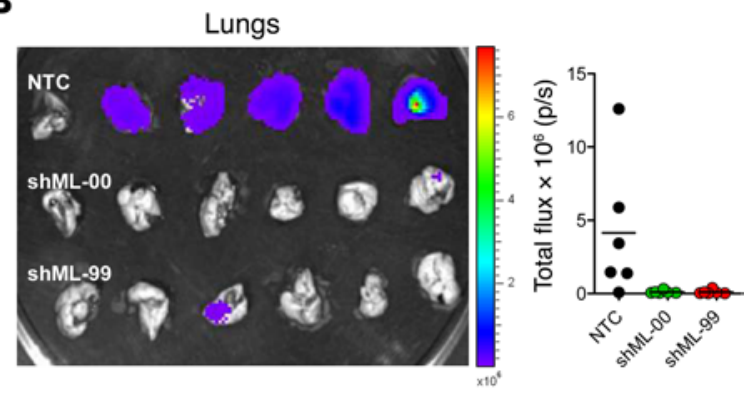

shML-99

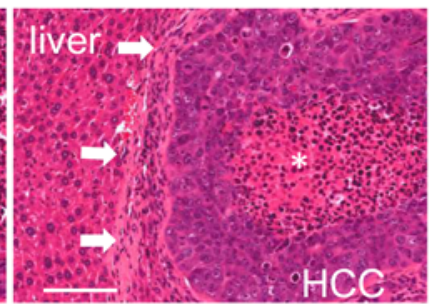

$\mathbf{F}$

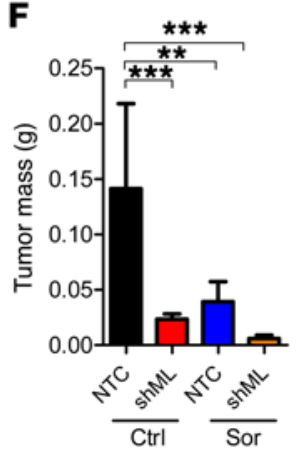

D

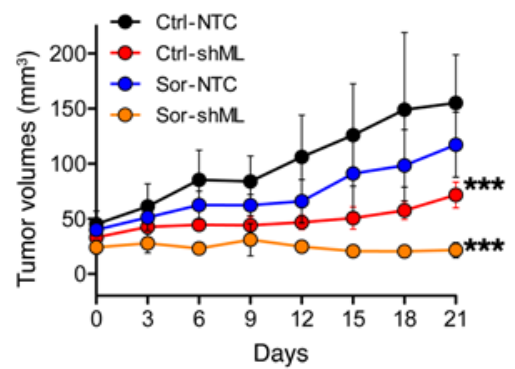

Figure 8. Knockdown of MTHFD1L suppressed HCC growth and sensitized HCC cells to sorafenib treatment in vivo. (A) Luciferase-labeled MHCC97L-NTC, -shML-00, and -shML-99 cells were orthotopically injected into nude mice ( $n=6$ mice per experimental group) and allowed to grow for 45 days. Left: Bioluminescent image of mice implanted with different stable cells. Middle: Representative picture of orthotopic xenografts. Right: Quantification of tumor size. (B) Lung tissues were removed from mice of the orthotopic liver implantation model for Xenogen imaging. Left: Bioluminescent image of lung tissues. Right: Quantification of luciferase intensities of HCC cells in lung tissues. (C) H\&E staining of the orthotopic tumors showing HCC-liver boundaries as indicated by arrows. Necrotic areas are represented by asterisks. (D) Growth curves of subcutaneous xenografts derived from MHCC97L-NTC and -shML-99 cells in nude mice that were administered vehicle control (Ctrl) or $30 \mathrm{mg} / \mathrm{kg} / \mathrm{d}$ sorafenib (Sor) ( $n=6$ mice per experimental group). (E) Representative pictures of subcutaneous xenografts. (F) Quantification of tumor mass. Error bars indicate mean $\pm \mathrm{SEM}$. ${ }^{* *} P<0.01,{ }^{* *} P<0.001 \mathrm{vs}$. NTC, Ctrl-NTC, or as indicated. 1 -way ANOVA. A and E: scale bar, $1 \mathrm{~cm}$; C: scale bar, $100 \mu \mathrm{m}$.

is retained. Our report provides clear evidence that cancer cells rely on folate-derived NADPH. Consistent with the computational analysis predicting that the largest proportion of NADPH ( $40 \%)$ in human proliferating cells comes from the folate cycle (8), our study confirms that the folate cycle plays a major role in oxidative stress defense in cancer while introducing an essential metabolic pathway for cancer growth.

Initiation of the folate cycle begins when serine reacts with THF. Serine can be either taken up exogenously or synthesized de novo from glucose, the latter suggesting a connection between the folate cycle and glucose metabolic pathways. G3P derived from glucose through glycolysis is converted to 3-phosphoglycerate, which is later converted to serine through 3 enzymes: phosphoglycerate dehydrogenase (PHGDH), phosphoserine aminotransferase (PSAT), and phosphoserine phosphatase (PSPH). A recent study showed that all the 3 serine biosynthetic enzymes were induced by c-MYC under nutrient starvation and were crucial to HCC growth (40). Our metabolomics data confirmed that serine accumulated upon knockdown of MTHFD1L, suggesting that the folate cycle indeed coordinates with serine and glucose metabolism to support cancer growth. A recent study showed that ATF4 bound with NRF2 to transcriptionally activate $P H G D H, P S A T$, and $P S P H$ (41). It is possible that MTHFD1L knockdown elevated ROS, which further stabilized NRF2, leading to the ATF4-associated activation of serine biosynthetic enzymes and subsequently serine accumulation. Another study showed that mTORC activated ATF4 for the transcription of MTHFD2 (42). The transcriptional program of ATF4 in HCC and its roles in HCC metabolism will be further explored in the future.

Interestingly, serine and glycine synthesis results in the production of glutathione, a tripeptide of glycine, glutamate, and cysteine (43). It was thus expectedly reported that knockdown of 
Table 3. Aggressiveness of HCC tumors derived from MHCC97L-NTC, -shML-00, and -shML-99 cells

$\begin{array}{lc}\text { Mouse } & \text { Tumor growth fronts } \\ \text { NTC-1 } & \text { Irregular } \\ \text { NTC-2 } & \text { Irregular } \\ \text { NTC-3 } & \text { Irregular } \\ \text { NTC-4 } & \text { Irregular } \\ \text { NTC-5 } & \text { Irregular } \\ \text { NTC-6 } & \text { Irregular } \\ \text { shMTHFD1L-00-1 } & \text { Regular } \\ \text { shMTHFD1L-00-2 } & \text { Regular } \\ \text { shMTHFD1L-00-3 } & \text { Partial irregular } \\ \text { shMTHFD1L-00-4 } & \text { Regular } \\ \text { shMTHFD1L-00-5 } & \text { Regular (encapsulated) } \\ \text { shMTHFD1L-00-6 } & \text { No tumor } \\ \text { shMTHFD1L-99-1 } & \text { Partial irregular } \\ \text { shMTHFD1L-99-2 } & \text { Partial irregular } \\ \text { shMTHFD1L-99-3 } & \text { Irregular } \\ \text { shMTHFD1L-99-4 } & \text { Regular (encapsulated) } \\ \text { shMTHFD1L-99-5 } & \text { Regular (encapsulated) } \\ \text { shMTHFD1L-99-6 } & \text { Regular (encapsulated) }\end{array}$

PSPH in HCC cells reduced glutathione production, which consequently induced oxidative stress (40). Our current study indicated that this elevation of oxidative stress might be related to the decline of serine entering the folate cycle, which would otherwise produce NADPH directly. Interestingly, the final and rate-limiting enzyme of serine biosynthesis, PSPH, was significantly upregulated in human HCC (40). These reports echo in harmony with our findings of MTHFD1L overexpression in HCC patients, suggesting that 1-carbon metabolism provides metabolic and survival advantages to HCC cells.

Our study provides evidence that MTHFD1L was regulated by the NRF2 pathway (44). The KEAP1/NRF2 pathway is the key pathway providing defense against oxidative stress and is one of the most frequently mutated pathways in human HCC (44). Recently demonstrated in a hepatocarcinogen-induced HCC mouse model, NRF2 mutations were found to be present through all stages of HCC and could be detected in the majority of early preneoplastic lesions (45). Intriguingly, these mutations were found to be missense and located in the KEAP1-binding region (45). As KEAP1 negatively regulates NRF2 expression through ubiquitin-proteosomal degradation, NRF2 mutations might result in stabilization of NRF2 that in turn activates antioxidant genes in HCC. TCGA data, however, revealed that KEAP1 and NRF2 mutations were found in only $4 \%$ and $3 \%$ of HCC samples, respectively (Supplemental Figure 7). These mutations were not significantly correlated with high MTHFD1L expression in HCC patients (Supplemental Figure 7). A possible explanation for the extensive KEAP1/ NRF2 mutations in mouse models is that the hepatocarcinogen might have created DNA damage in the mouse livers. KEAP1/ NRF2 mutations should be further validated in other cohorts of HCC patients in the future. Functional characterization studies to directly and precisely elucidate which particular KEAP1 and NRF2 mutations affect MTHFD1L expression and confer resistance to oxidative stress on HCC cells are also awaited.

Apart from NADPH, the folate cycle generates purine and pyrimidine; however, reduction of nucleotides in MTHFD1Lknockdown HCC cells was not observed, despite a significant decrease in cancer growth. This is similar to our previous observation in PPP blockade (6). PPP is connected to glycolysis and is a major pathway that produces NADPH and ribose 5-phosphate (R5P), the backbone of DNA. Suppression of PPP in HCC through inhibition of transketolase reduced NADPH with no significant reduction in R5P but strongly inhibited HCC growth (6). Our previous study together with this current one strongly supports the notion that cancer cells have greater demands for antioxidants than building blocks to support primary cancer growth. A worthwhile study recently demonstrated that the folate cycle allowed melanoma cells to survive within the circulation and metastasize distantly (25). Our orthotopic liver implantation model has shown that the knockdown of MTHFD1L prominently reduced lung metastasis. However, it is unclear whether it was a secondary response due to reduced primary tumor growth. Metastatic efficiency of cancer cells could be further evaluated by tail-vein or splenic injection in the future.

Recently, Ducker et al. elegantly demonstrated that cells would compensate for the deficiency in the mitochondrial folate pathway by reversing the cytosolic 1C flux, suggesting flexibilities between the mitochondrial and cytosolic compartments of the folate cycle (24). Ducker et al. showed that blocking the mitochondrial folate pathway by knocking out MTHFD2, MTHFD1L, or SHMT2 using the CRISPR-Cas9 genome editing approach in cells inhibited the generation of 10-CHO-THF, making cells dependent on SHMT1 for $1 \mathrm{C}$ unit production. They further showed that blocking SHMT1 and MTHFD2 together could completely retard colorectal cancer growth. This important study provided an explanation as to why MTHFD1L knockdown or knockout alone could not achieve full HCC growth inhibition, as only the mitochondrial compartment is blocked. This might also explain why the effects of knockout are not as dramatic as those of knockdown, because MTHFD1Lknockout cells might potentially be even more dependent on the cytoplasmic compartment than MTHFD1L-knockdown cells. A point worth mentioning is that NADPH from the mitochondrial compartment of the folate cycle is mainly produced by ALDH1L2, which competes with MTHFD1L for 10-CHO-THF. However, MTHFD1L is required for the production of formate, which could pass through the mitochondrial membrane. We further interrogated the ratios of these 2 enzymes in nontumorous and HCC tissues from the TCGA database. We found the MTHFD1L/ALDH1L2 ratio elevated in HCC (Supplemental Figure 8), suggesting that more 10-CHO-THF might be used by MTHFD1L to generate formate, which could sustain the continuity of the cytoplasmic compartment of the folate cycle in HCC. Another interesting point to note in the mitochondrial compartment of the folate cycle is that MTHFD2 and MTHFD2L compete for $\mathrm{CH}_{2}$-THF to make NADH and NADPH, respectively. TCGA data revealed that the mean values of the mRNA expression levels of MTHFD2 and MTHFD2L in HCC were 143.10 and 156.70 , respectively (data not shown), suggesting that both enzymes are robustly expressed and both NADH and NADPH are required for HCC growth. The competi- 
A

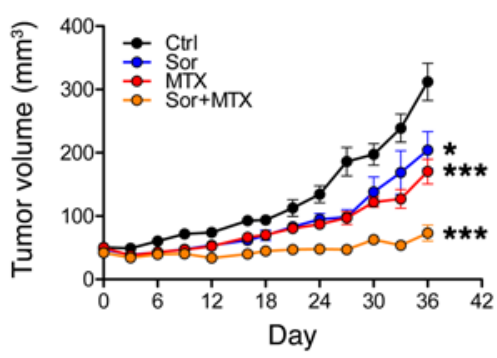

D

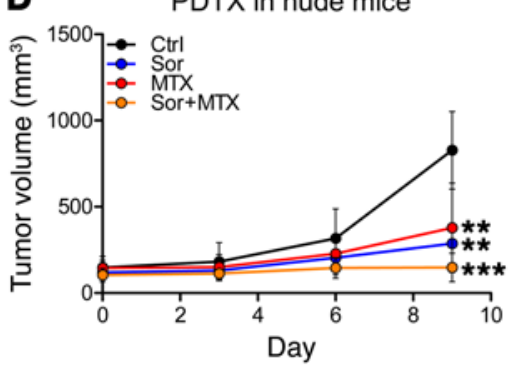

B

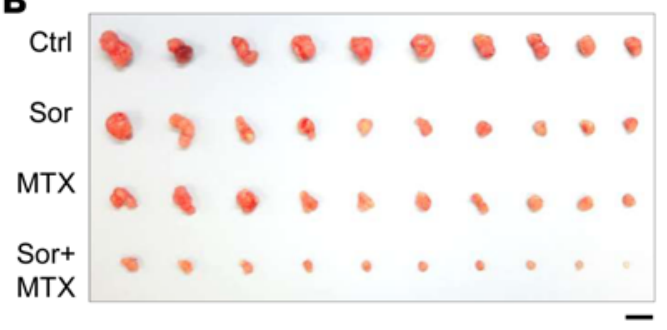

E

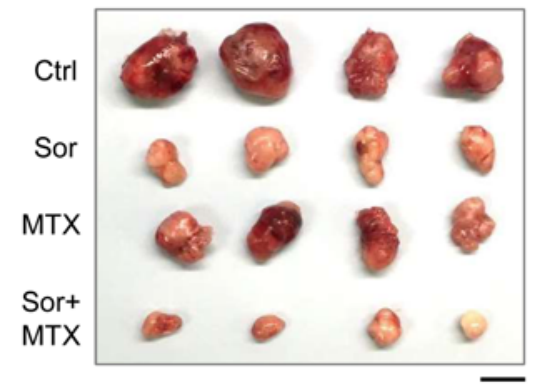

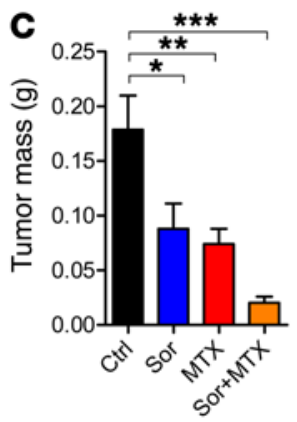

$\mathbf{F}$

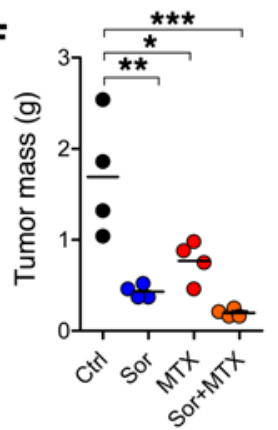

G

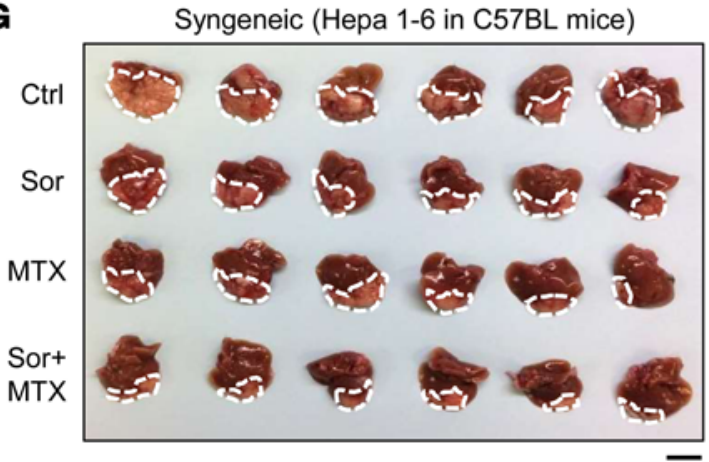

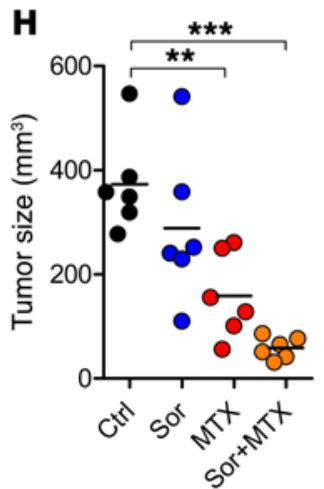

Figure 9. Inhibition of the folate cycle suppressed HCC growth and sensitized HCC cells to sorafenib treatment in vivo. (A) Growth curves of subcutaneous xenografts derived from MHCC97L HCC cells in nude mice that were administered vehicle control (Ctrl), $30 \mathrm{mg} / \mathrm{kg} / \mathrm{d}$ sorafenib (Sor), or $1.5 \mathrm{mg} / \mathrm{kg} / \mathrm{d}$ MTX, or coadministered both $30 \mathrm{mg} / \mathrm{kg} / \mathrm{d}$ sorafenib and $1.5 \mathrm{mg} / \mathrm{kg} / \mathrm{d}$ MTX (Sor+MTX) ( $n=10$ mice per experimental group). (B) Representative pictures of subcutaneous xenografts. (C) Quantification of tumor mass. (D) Growth curves of subcutaneous xenografts from patient-derived tumor xenograft (PDTX, PY003) in nude mice that were administered vehicle control, $30 \mathrm{mg} / \mathrm{kg} / \mathrm{d}$ sorafenib, or $1.5 \mathrm{mg} / \mathrm{kg} / \mathrm{d}$ MTX, or coadministered both $30 \mathrm{mg} / \mathrm{kg} / \mathrm{d}$ sorafenib and $1.5 \mathrm{mg} / \mathrm{kg} / \mathrm{d}$ MTX (Sor+MTX) ( $n=4$ mice per experimental group). (E) Representative pictures of subcutaneous xenografts. (F) Quantification of tumor mass. (G) Representative photo of tumors orthotopically injected with mouse Hepa 1-6 HCC cells in C57BL/6N immune-competent mice (syngeneic) administered either vehicle control, sorafenib (30 mg/kg/d), MTX (3 mg/kg/d), or a combination of both (Sor+MTX) for 7 days ( $n=6$ mice per experimental group). (H) Quantification of the tumor size. Error bars indicate mean \pm SEM. ${ }^{*} P<0.05,{ }^{* *} P<0.01,{ }^{* * *} P<0.001$ vs. Ctrl, or as indicated. 1-way ANOVA. B, E, and G: scale bar: $1 \mathrm{~cm}$.

tion between MTHFD1L and ALDH1L2 as well as MTHFD2 and MTHFD2L in the folate cycle will be an exciting avenue to be investigated.

The human body cannot synthesize folate de novo; thus folate must be taken up from diets. Dietary folate has long been advocated as having anticancer effects. Earlier epidemiological studies showed that dietary folate reduced the risk of several cancers, including breast and colorectal cancer $(46,47)$. These earlier studies were coherent with the traditional belief that ROS are protumorigenic. This long-standing concept, however, has been shaken by our renewed attention and expanding knowledge on cancer metabolism. A recent study showed that diet supplemented with antioxidant vitamin E or NAC promoted cancer develop- ment in B-RAF- and K-RAS-associated lung cancers in mice (48). It was also shown that administration of the antioxidant NAC promoted melanoma cell metastasis (25). However, the extent and stage of diseases at which dietary folate or other antioxidant intakes are deemed harmful to human health remain unclear. On the other hand, it has become apparent that antioxidantproducing pathways are attractive therapeutic targets for cancer cells given their increased reliance on antioxidants. Recently, an elegant study showed that combined usage of glutathione and thioredoxin inhibitors could efficiently block cancer initiation and growth in breast cancer model (5). Our current study has unequivocally showed that the inhibition of the folate cycle rendered cancer cells more sensitive to treatment with sorafenib, 
the only FDA-approved targeted therapeutic agent for advanced HCC patients. As we show that MTHFD1L is overexpressed in multiple solid cancers, it will be appealing to investigate whether folate inhibitors could be used in combination with other existing therapies in other cancer types. As we begin to understand more about the metabolic vulnerabilities in cancer, development of more precise inhibitors that specifically target the associated molecules is under way.

\section{Methods}

Clinicopathological correlation analysis and survival analysis. Clinicopathological features of human HCC were correlated with MTHFD1L expression in clinical specimens. Correlation of the features, including cellular differentiation, direct liver invasion, hepatitis B surface antigen, tumor encapsulation, tumor microsatellite formation, tumor size, and venous invasion, was performed using SPSS 20.0 software (IBM Corp.). Pathological tumor node metastasis (pTNM) tumor staging was analyzed and graded by a pathologist. Demographics of HCC patients from our center are summarized in Supplemental Table 1. Patient survival data of 442 HCC patients were obtained from TCGA cBioPortal. MTHFD1L expression in HCC patients was defined as high if the $Z$ score was greater than 1, or otherwise low. Survival analysis was determined by Kaplan-Meier followed by log-rank test.

Cell culture. HCC cell lines MHCC97L, PLC/PRF/5, Huh7, HepG2, Hep3B, and Hepa 1-6 were used. MHCC97L was a gift from Z.Y. Yang (Fudan University of Shanghai, Shanghai, China). HepG2, Hep3B, and Hepa 1-6 were obtained from American Type Culture Collection (ATCC) and were cultured in DMEM-high glucose (DMEMHG) media supplemented with sodium pyruvate, unless indicated. PLC/PRF/5 and Huh7, both obtained from ATCC, were cultured with DMEM-HG, unless indicated. All culture media were supplemented with 10\% FBS (all reagents from Gibco).

RNA extraction, reverse transcription $P C R$, and quantitative realtime PCR. Total RNA was extracted from clinical specimens that were snap-frozen using liquid nitrogen prior to storage at $-80^{\circ} \mathrm{C}$, and HCC cell lines using the TRIzol reagent (Life Technologies). cDNA was synthesized from purified RNA samples by reverse transcription using the GeneAmp PCR Reagent Kit (Applied Biosystems). Quantitative realtime PCR amplifications of MTHFD1L and the internal control 18S were performed using the TaqMan Gene Expression Assay (Applied Biosystems) for clinical specimens and using the SYBR Green qPCR Master Mix (Applied Biosystems) with corresponding primers (Supplemental Table 2) for HCC cell lines.

Transcriptome sequencing. Transcriptome sequencing was performed using 16 pairs of human HCC tissues and their adjacent nontumorous liver tissues. The poly $(\mathrm{A})^{+}$mRNA library was prepared using the TruSeq Stranded mRNA Sample Prep Kit (Illumina). Using Illumina HiSeq2000 (Axeq Technologies), 100-bp paired-end sequencing was performed. Subsequent data analysis was performed by the TopHat-Cufflinks pipeline, and the resulting values were indicated by FPKM (fragments per kilobase of transcript sequence per million mapped reads).

The Cancer Genome Atlas. Transcriptome sequencing data using 50 pairs of human HCC tissues and their adjacent nontumorous liver tissues as well as other solid cancers including esophageal cancer, gastric adenocarcinoma, bladder cancer, lung adenocarcinoma, breast cancer, colon adenocarcinoma, kidney renal cell carcinoma, and pros- tate cancer were retrieved from The Cancer Genome Atlas (TCGA) via the Broad GDAC Firehose (Broad Institute). Only the available paired samples with both solid tumors and nontumorous counterparts were included in the comparison study. Cases without matched nontumorous tissues were excluded for this analysis. Comparisons were done with paired $t$ test.

ChIP assay. ChIP assay was performed as we previously described (49). In brief, cells were fixed with $3.7 \%$ formalin (vol/vol) and then sonicated. Sheared DNA was incubated with antibodies against NRF2 (anti-rabbit, catalog ab62352, Abcam) or IgG control (anti-rabbit, catalog sc-2027, Santa Cruz Biotechnology). DNA-protein-antibody complexes were incubated with Protein A/Salmon Sperm DNA Agarose Beads (Thermo Fisher Scientific). Beads were washed sequentially with gradients of salt buffer and eluted in $1 \% \mathrm{SDS} / \mathrm{NaHCO}_{3}$. ChIP DNA was analyzed with primers flanking the putative AREs. Primer sequences are provided in Supplemental Table 2.

Stable HCC cell establishment. HCC cell lines with stable knockdowns were produced using a lentiviral-mediated approach as previously described (49-51). In short, pLKO.1-puro vectors encompassing shRNAs targeting MTHFD1L (shMTHFD1L), NRF2 (shNRF2), or a nontarget control were stably transfected into HCC cell lines. Puromycin was used for the selection of HCC cells with stable expression of shRNAs. MTHFD1L- and NRF2-overexpressing cell lines were generated using CRISPR-dCas9 Synergistic Activation Mediator (SAM) system as previously described (52). dCas9-VP64, MS2-p65HSF1, and single guide RNAs (sgRNAs) targeting the promoters of MTHFD1L were transfected into MHCC97L cells with sequential lentiviral infections. Zeocin was used for the selection of HCC cells with stable expression of sgRNAs for the CRISPR-dCas9 SAM system. For MTHFD1L-knockout cell establishment, Cas9 and sgRNA were introduced into MHCC97L and PLC cells through a lentiviral-mediated approach as previously described (53). Puromycin was used for the selection of HCC cells with stable expression of the sgRNAs for the CRISPR-Cas9 gene editing (KO) system. Sequences of all sgRNAs and shRNAs are provided in Supplemental Table 3.

Western blotting. Protein extraction was performed on clinical HCC specimens snap-frozen using liquid nitrogen before storage at $-80^{\circ} \mathrm{C}$, and on HCC cells using the radioimmunoprecipitation assay (RIPA) buffer supplemented with complete protease inhibitor (Roche) and PhosSTOP phosphatase inhibitor cocktails (Roche). Equal volumes of protein lysates were separated using 10\% (vol/vol) SDSPAGE, and transferred onto PVDF membranes (GE Healthcare). For immunoblotting, antibodies against MTHFD1L (anti-rabbit, catalog HPA029041, Abcam and Atlas Antibodies), NDUFA4 (anti-rabbit, catalog ab129752, Abcam), NRF2 (anti-rabbit, catalog 12721, Cell Signaling Technology), and $\beta$-actin (anti-mouse, catalog A5316, SigmaAldrich) were used.

Cell proliferation assays. Cell proliferation rates were measured using 2 different assays: cell counting and 5-bromo-2'-deoxyuridine (BrdU) colorimetric (Roche) assay. For proliferation rates determined by cell counting, $2 \times 10^{4}$ cells per well were plated onto 12 -well culture plates. Cells were trypsinized and counted using the Z1 Particle Counter (Beckman Coulter) 24 hours after plating of cells, daily for 4 days. For proliferation rates determined by BrdU assay, $1 \times 10^{4}$ cells per well were plated onto 96-well culture plates. BrdU labeling and colorimetric measurements were performed 24 hours after plating of cells according to the manufacturer's instructions (Roche). 
ROS, NADPH, and 8-OH-dG measurements. Intracellular ROS levels were measured in equal amounts of HCC cells plated into 6-well dishes. Cells were trypsinized and washed with PBS before staining with $2 \mu \mathrm{mol} / 1$ chloromethyl-20,70-dichlorodihydrofluorescein diacetate (CM- $\left.\mathrm{H}_{2} \mathrm{DCFDA}\right)$ oxidative stress indicator (Life Technologies) and analyzed using the FACSCanto II Analyzer (BD Biosciences) flow cytometer. Results were analyzed using FlowJo software (FlowJo). Intracellular $\mathrm{NADPH} / \mathrm{NADP}^{+}$ratios were determined in $\mathrm{HCC}$ cells using the NADP/NADPH Quantification Colorimetric Kit (BioVision) according to the manufacturer's instructions. Briefly, equal amounts of plated HCC cells on 6-well dishes were trypsinized and subjected to extraction by 2 freeze/thaw cycles using the Extraction Buffer. Portions of the extracted samples were heated at $60^{\circ} \mathrm{C}$ for 30 minutes to allow for NADP decomposition while the other portion was left on ice (unheated). Both portions of the sample then underwent NADP cycling through incubation with the Cycling Buffer for 5 minutes before reaction development with the NADPH Developer. Intensities of the NADPH and NAPD signals were measured with a plate reader at an $\mathrm{OD}$ of $450 \mathrm{~nm}$. The $\mathrm{NADPH} / \mathrm{NADP}^{+}$ratio was calculated as: (intensity of heated samples) / (intensity of unheated samples intensity of heated samples). For 8-OH-dG assay, $5 \times 10^{4}$ MHCC97LNTC or -shMTHFD1L cells were seeded on 12-well plates, and DNA was extracted by phenol chloroform. Seven micrograms of DNA was added into an 8-OH-dG antibody-precoated ELISA plate (Abcam) and incubated at room temperature for 1 hour. The ELISA plate was subsequently washed and developed with substrates following the manufacturer's protocol (Abcam).

Oxygen consumption rate analysis. Oxygen consumption was measured by XFp Cell Mito Stress Test according to the manufacturer's protocol (Seahorse Bioscience). In brief, MHCC97L-NTC or-shMTHFD1L cells were seeded onto miniplates at $50 \%$ confluence. One micromolar oligomycin, 0.125-2 $\mu \mathrm{M}$ FCCP, and $0.5 \mu \mathrm{M}$ rotenone and antimycin A were injected into wells at the indicated time points, and oxygen content was measured by XFp Analyzer (Seahorse Bioscience).

Cell cycle analysis. The analysis of cell cycle distributions was performed using propidium iodide (PI) stain (Calbiochem) subjected to flow cytometric analysis. Briefly, cells were treated with nocodazole (200 ng/ml) for 16 hours for synchronization in the G2/M phase of the cell cycle. Nocodazole was removed from the cells after synchronization for 9 hours before harvesting for fixation using $75 \%$ (vol/vol) ethanol for 1 hour. Cells were stained with $50 \mu \mathrm{g} / \mathrm{ml}$ PI for 30 minutes under incubation before analysis using the FACSCanto II Analyzer (BD Biosciences).

Cell apoptosis analysis. Cells were cultured in serum-free medium for 24 hours before trypsinization and subsequently resuspended in Annexin V binding buffer (BD Biosciences) with annexin V-FITC (MBL International) and PI (BD Biosciences) for staining at room temperature for 15 minutes according to the manufacturer's instructions. The percentage of cell apoptosis for each sample was analyzed with flow cytometry.

Metabolomics. MHCC97N-NTC or-shMTHFD1L cells were washed with $5 \%$ (wt/wt) mannitol and scraped with methanol in the presence of $10 \mu \mathrm{M}$ of internal control solution comprising compound $\mathrm{C} 1$ with $\mathrm{m} / z$ at 182.048 and compound A1 with $\mathrm{m} / z$ at 231.070 (Human Metabolome Technologies). Supernatants were filtered by centrifuge filter unit and were evaporated by centrifugation. Extracted intracellular metabolites were analyzed by CE-TOFMS by Human Metabolome Technologies.
Animal experiments. Animal experiments were performed on 6- to 8-week-old male BALB/cAnN-nu (nude) mice. For the orthotopic tumor model, $1 \times 10^{6} \mathrm{MHCC} 97 \mathrm{~L}$ cells, luciferase-labeled, were resuspended in $100 \%$ Matrigel (BD Biosciences) and injected into the left lobes of the livers of the nude mice. Drug administration began 1 week after inoculation. At 6 weeks (42 days) after inoculation, tumor-bearing mice were administered $100 \mathrm{mg} / \mathrm{kg}$ D-Luciferin (PerkinElmer) via i.p. injections for bioluminescent imaging using the Xenogen IVIS 100 Imaging System (Xenogen). Livers and lungs were also harvested for ex vivo imaging as well as $10 \%$ formalin fixation followed by H\&E staining for histological analysis. For the syngeneic model, $4 \times 10^{6} \mathrm{Hepa}$ 1-6 cells were resuspended in $100 \%$ Matrigel and injected into the left lobes of the livers of 4- to 6-week-old male C57BL/6N mice. Four days after inoculation, tumor-bearing mice were administered sorafenib, MTX, both, or vehicle controls for 7 days. Livers were harvested, and tumor volumes were measured using electronic caliper and calculated using the following formula: tumor volume $=$ length $(\mathrm{mm}) \times$ width $(\mathrm{mm}) \times$ height $(\mathrm{mm})$ $\times$ 0.52. For subcutaneous tumor models, $1 \times 10^{6}$ MHCC97L cells were resuspended in $100 \mu \mathrm{l}$ PBS and injected s.c. onto either flank of the nude mice. Tumor volumes were measured using an electronic caliper and calculated as mentioned above. Drug administration began 1 week after inoculation. For the patient-derived tumor xenograft model, $1 \times 10^{6} \mathrm{cells}$ from an HCC patient (PYO03) were resuspended in 100\% Matrigel and injected s.c. into nude mice. Drug administration began 11 days after inoculation. Tumor volumes were measured using an electronic caliper and calculated as mentioned above.

Pharmacological studies. HCC cells were treated with pharmacological agents in vitro for cell proliferation assay, ROS measurement, cell cycle analysis, and cell apoptosis experiments. Sorafenib (LC Laboratories) was dissolved in DMSO, while MTX (Sigma-Aldrich) was dissolved in $\mathrm{H}_{2} \mathrm{O}$ and $0.5 \% 4 \mathrm{M} \mathrm{NaOH}$, and glutathione reduced ethyl ester (GSH-EE) (Cayman Chemical), L-glutathione reduced (GSH), and $\mathrm{N}$-acetyl-L-cysteine (NAC) (both from Sigma-Aldrich) were all dissolved in $\mathrm{H}_{2} \mathrm{O}$. Sorafenib and MTX were also administered via oral gavage and i.p. injections, respectively, in vivo daily.

Oil Red $O$ staining. Staining of neutral triglyceride and lipids was performed on cultured cells and frozen sections from livers of HCCbearing mice using Oil Red O (Sigma-Aldrich) dissolved in isopropanol. To stain cultured cells, $1 \times 10^{5}$ MHCC97L-NTC or -shML cells were seeded onto 6-well plates. Plated cells were fixed with $10 \%$ formalin before staining with Oil Red O working solution diluted with distilled water. After removal of the stain and several washes with distilled water, Oil Red O dye on the dried cells was eluted with $100 \%$ isopropanol. Relative amounts of neutral triglyceride and lipids were determined by OD measurements at $500 \mathrm{~nm}$ with $100 \%$ isopropanol as blank. To stain for neutral triglyceride and lipids from livers of HCC-bearing mice, frozen sections were air-dried and fixed in formalin. After rinsing with $60 \%$ isopropanol, frozen sections were stained in Oil Red O before another rinse with 60\% isopropanol. Sections were stained with hematoxylin, rinsed with hard water, and finally mounted in aqueous mountant for imaging and microscope analysis.

Statistics. All statistical analyses were performed using GraphPad Prism 5.0 software (GraphPad Software Inc.) by either 1-way ANOVA with Bonferroni's correction, Student's $t$ test, or Wilcoxon signed-rank test. All functional assay experiments are representations of at least 3 independent experiments expressed as mean \pm SEM. Statistical significance was considered with a $P$ value less than 0.05 . 
Study approval. The use of clinical HCC tumor samples and their adjacent nontumorous liver tissues in the study was approved by the Institutional Review Board of the University of Hong Kong and the Hospital Authority of Hong Kong (HKU/HA HKW IRB; ref. no. UW 09-158). The clinical specimens were obtained from patients admitted to Queen Mary Hospital. Signed consent forms were obtained from patients as acknowledgment prior to the collection and usage of resected tissues. All experimental procedures performed on animals were approved by the Committee on the Use of Live Animals in Teaching and Research of the University of Hong Kong and conformed to the Animals (Control of Experiments) Ordinance of Hong Kong. All animal experiments were performed under the UK Co-ordinating Committee on Cancer Research PMID: 9459138 Guidelines for the Welfare of Animals in Experimental Neoplasia to ensure minimal suffering during the experimental procedures (54).

\section{Author contributions}

DL, IOLN, and CCLW designed the studies. DL and CCLW wrote the paper. DL, IMJX, DKCC, RKHL, APWT, LLL, CTL, FHCT, LLW, CYKC, CMW, and CCLW performed the experiments. CMW and CCLW performed statistical analysis.

\section{Acknowledgments}

We thank the Faculty Core Facility of the University of Hong Kong Li Ka Shing Faculty of Medicine for their technical support in flow cytometry analysis and animal imaging. We thank the Laboratory Animal Unit of the University of Hong Kong for supporting our animal work. We thank the State Key Laboratory of Pharmaceutical Biotechnology, University of Hong Kong (Aiming Xu and Kenneth Cheung), for their advice and technical support in the Seahorse XFp Cell Mito Stress Test. Carmen Chak-Lui Wong is a recipient of the Croucher Innovation Award. This work was funded by the Research Grant Council General Research Fund (grant 17153116) and Theme-Based Research Scheme (T12-704/16-R).

Address correspondence to: Carmen Chak-Lui Wong, Department of Pathology, The University of Hong Kong, L7-04, Laboratory Block, 21 Sassoon Road, Pokfulam, Hong Kong, China. Phone: 852.2255.5077; E-mail: carmencl@pathology.hku.hk. Or to: Irene Oi-Lin Ng, Department of Pathology, The University of Hong Kong, Room 315, University Pathology Building, Queen Mary Hospital, Pokfulam, Hong Kong, China. Phone: 852.2255.4882; E-mail: iolng@hku.hk.
1. Locasale JW, Cantley LC. Metabolic flux and the regulation of mammalian cell growth. Cell Metab. 2011;14(4):443-451.

2. Szatrowski TP, Nathan CF. Production of large amounts of hydrogen peroxide by human tumor cells. Cancer Res. 1991;51(3):794-798.

3. Sena LA, Chandel NS. Physiological roles of mitochondrial reactive oxygen species. Mol Cell. 2012;48(2):158-167.

4. Metallo CM, Vander Heiden MG. Understanding metabolic regulation and its influence on cell physiology. Mol Cell. 2013;49(3):388-398.

5. Harris IS, et al. Glutathione and thioredoxin antioxidant pathways synergize to drive cancer initiation and progression. Cancer Cell. 2015;27(2):211-222.

6. Xu IM, et al. Transketolase counteracts oxidative stress to drive cancer development. Proc Natl Acad Sci U S A. 2016;113(6):E725-E734.

7. Renschler MF. The emerging role of reactive oxygen species in cancer therapy. Eur J Cancer. 2004;40(13):1934-1940.

8. Fan J, Ye J, Kamphorst JJ, Shlomi T, Thompson CB, Rabinowitz JD. Quantitative flux analysis reveals folate-dependent NADPH production. Nature. 2014;510(7504):298-302.

9. Pike ST, Rajendra R, Artzt K, Appling DR. Mitochondrial C1-tetrahydrofolate synthase (MTHFD1L) supports the flow of mitochondrial one-carbon units into the methyl cycle in embryos. J Biol Chem. 2010;285(7):4612-4620.

10. Tibbetts AS, Appling DR. Compartmentalization of Mammalian folate-mediated one-carbon metabolism. Annu Rev Nutr. 2010;30:57-81.

11. Farber S. Some observations on the effect of folic acid antagonists on acute leukemia and other forms of incurable cancer. Blood. 1949;4(2):160-167.

12. Momb J, et al. Deletion of Mthfd1l causes embryonic lethality and neural tube and craniofacial defects in mice. Proc Natl Acad Sci U S A. 2013;110(2):549-554.
13. Naj AC, et al. Dementia revealed: novel chromosome 6 locus for late-onset Alzheimer disease provides genetic evidence for folate-pathway abnormalities. PLoS Genet. 2010;6(9):e1001130.

14. Parle-McDermott A, et al. A common variant in MTHFD1L is associated with neural tube defects and mRNA splicing efficiency. Hum Mutat. 2009;30(12):1650-1656.

15. Samani NJ, et al. Genomewide association analysis of coronary artery disease. $N$ Engl JMed. 2007;357(5):443-453.

16. Jain M, et al. Metabolite profiling identifies a key role for glycine in rapid cancer cell proliferation. Science. 2012;336(6084):1040-1044.

17. Sugiura T, Nagano Y, Inoue T, Hirotani K. A novel mitochondrial C1-tetrahydrofolate synthetase is upregulated in human colon adenocarcinoma. Biochem Biophys Res Commun . 2004;315(1):204-211.

18. Selcuklu SD, et al. MicroRNA-9 inhibition of cell proliferation and identification of novel miR-9 targets by transcriptome profiling in breast cancer cells. J Biol Chem. 2012;287(35):29516-29528.

19. Minguzzi S, Selcuklu SD, Spillane C, ParleMcDermott A. An NTD-associated polymorphism in the 3' UTR of MTHFD1L can affect disease risk by altering miRNA binding. Hum Mutat. 2014;35(1):96-104.

20. Nilsson R, et al. Metabolic enzyme expression highlights a key role for MTHFD2 and the mitochondrial folate pathway in cancer. Nat Commun. 2014;5:3128.

21. Lee GY, et al. Comparative oncogenomics identifies PSMB 4 and SHMT2 as potential cancer driver genes. Cancer Res. 2014;74(11):3114-3126.

22. Locasale JW. Serine, glycine and one-carbon units: cancer metabolism in full circle. Nat Rev Cancer. 2013;13(8):572-583.

23. Yang M, Vousden KH. Serine and one-carbon metabolism in cancer. Nat Rev Cancer. 2016;16(10):650-662.

24. Ducker GS, et al. Reversal of cytosolic one-carbon flux compensates for loss of the mitochondria folate pathway. Cell Metab. 2016;24(4):640-641.

25. Piskounova E, et al. Oxidative stress inhibits distant metastasis by human melanoma cells. Nature. 2015;527(7577):186-191.

26. Cheng AL, et al. Efficacy and safety of sorafenib in patients in the Asia-Pacific region with advanced hepatocellular carcinoma: a phase III randomised, double-blind, placebo-controlled trial. Lancet Oncol. 2009;10(1):25-34.

27. Llovet JM, et al. Sorafenib in advanced hepatocellular carcinoma. N Engl JMed. 2008;359(4):378-390.

28. Mitsuishi Y, et al. Nrf2 redirects glucose and glutamine into anabolic pathways in metabolic reprogramming. Cancer Cell. 2012;22(1):66-79.

29. Wasserman WW, Fahl WE. Functional antioxidant responsive elements. Proc Natl Acad SciUS A. 1997;94(10):5361-5366.

30. Björnson E, et al. Stratification of Hepatocellular Carcinoma Patients Based on Acetate Utilization. Cell Rep. 2015;13(9):2014-2026.

31. Calo N, et al. Stress-activated miR-21/miR-21* in hepatocytes promotes lipid glucose metabolic disorders associated with high-fat diet consumption. Gut. 2016;65(11):1871-1881.

32. Li L, et al. Differential requirement for de novo lipogenesis in cholangiocarcinoma and hepatocellular carcinoma of mice and humans. Hepatology. 2016;63(6):1900-1913.

33. Hong $\mathrm{X}$, et al. PTEN antagonises Tcl1/hnRNPKmediated G6PD pre-mRNA splicing which contributes to hepatocarcinogenesis. Gut. 2014;63(10):1635-1647.

34 . Wen D, et al. Malic enzyme 1 induces epithelialmesenchymal transition and indicates poor prognosis in hepatocellular carcinoma. Tumour Biol. 2015;36(8):6211-6221.

35. Jiang P, Du W, Mancuso A, Wellen KE, Yang X. Reciprocal regulation of p53 and malic enzymes modulates metabolism and senescence. Nature. 2013;493(7434):689-693. 
36. Ward PS, et al. The common feature of leukemiaassociated IDH1 and IDH2 mutations is a neomorphic enzyme activity converting alphaketoglutarate to 2-hydroxyglutarate. Cancer Cell. 2010;17(3):225-234.

37. Xu W, et al. Oncometabolite 2-hydroxyglutarate is a competitive inhibitor of $\alpha$-ketoglutaratedependent dioxygenases. Cancer Cell. 2011;19(1):17-30.

38. Mardis ER, et al. Recurring mutations found by sequencing an acute myeloid leukemia genome. N Engl J Med. 2009;361(11):1058-1066.

39. Yan H, et al. IDH1 and IDH2 mutations in gliomas. N EnglJM Med. 2009;360(8):765-773.

40. Sun L, et al. cMyc-mediated activation of serine biosynthesis pathway is critical for cancer progression under nutrient deprivation conditions. Cell Res. 2015;25(4):429-444.

41. DeNicola GM, et al. NRF2 regulates serine biosynthesis in non-small cell lung cancer. Nat Genet. 2015;47(12):1475-1481.

42. Ben-Sahra I, Hoxhaj G, Ricoult SJ, Asara JM, Manning BD. mTORC1 induces purine synthesis through control of the mitochondrial tetrahydrofolate cycle. Science. 2016;351(6274):728-733.

43. Anderson ME. Glutathione: an overview of biosynthesis and modulation. Chem Biol Interact. 1998;111-112:1-14.

44. Schulze K, et al. Exome sequencing of hepatocellular carcinomas identifies new mutational signatures and potential therapeutic targets. Nat Genet. 2015;47(5):505-511.

45. Zavattari P, et al. Nrf2, but not $\beta$-catenin, mutation represents an early event in rat hepatocarcinogenesis. Hepatology. 2015;62(3):851-862.

46. Lamprecht SA, Lipkin M. Chemoprevention of colon cancer by calcium, vitamin D and folate: molecular mechanisms. Nat Rev Cancer. 2003;3(8):601-614.

47. Tio M, Andrici J, Cox MR, Eslick GD. Folate intake and the risk of upper gastrointestinal cancers: a systematic review and meta-analysis. J Gastroenterol Hepatol. 2014;29(2):250-258.

48. Sayin VI, Ibrahim MX, Larsson E, Nilsson JA, Lindahl P, Bergo MO. Antioxidants accelerate lung cancer progression in mice. Sci Transl Med. 2014;6(221):221ra15.

49. Wong CC, et al. Hypoxia-inducible factor 1 is a master regulator of breast cancer metastatic niche formation. Proc Natl Acad Sci U S A. 2011;108(39):16369-16374.

50. Wong CC, et al. Lysyl oxidase-like 2 is critical to tumor microenvironment and metastatic niche formation in hepatocellular carcinoma. Hepatology. 2014;60(5):1645-1658.

51. Wong CC, et al. The microRNA miR-139 suppresses metastasis and progression of hepatocellular carcinoma by down-regulating Rho-kinase 2. Gastroenterology. 2011;140(1):322-331.

52. Konermann S, et al. Genome-scale transcriptional activation by an engineered CRISPR-Cas 9 complex. Nature. 2015;517(7536):583-588.

53. Sanjana NE, Shalem O, Zhang F. Improved vectors and genome-wide libraries for CRISPR screening. Nat Methods. 2014;11(8):783-784.

54. United Kingdom Co-ordinating Committee on Cancer Research (UKCCCR) Guidelines for the Welfare of Animals in Experimental Neoplasia (Second Edition). Br JCancer. 1998;77(1):1-10. 